\title{
On Higgs decays to hadrons and the R-ratio at $\mathrm{N}^{4} \mathrm{LO}$
}

\author{
F. Herzog, ${ }^{a}$ B. Ruijl, ${ }^{a, b}$ T. Ueda, ${ }^{a}$ J.A.M. Vermaseren ${ }^{a}$ and A. Vogt $^{c}$ \\ ${ }^{a}$ Nikhef Theory Group, \\ Science Park 105, 1098 XG Amsterdam, The Netherlands \\ ${ }^{b}$ Leiden Centre of Data Science, Leiden University, \\ Niels Bohrweg 1, 2333 CA Leiden, The Netherlands \\ ${ }^{c}$ Department of Mathematical Sciences, University of Liverpool, \\ Liverpool L69 3BX, U.K. \\ E-mail: fherzog@nikhef.nl, benruyl@gmail.com, tueda@nikhef.nl, \\ t68@nikhef.nl, Andreas.Vogt@liverpool.ac.uk
}

ABSTRACT: We present the first determination of Higgs-boson decay to hadrons at the nextto-next-to-next-to-next-to-leading order of perturbative QCD in the limit of a heavy top quark and massless light flavours. This result has been obtained by computing the absorptive parts of the relevant five-loop self-energy for a general gauge group and combining the outcome with the corresponding coefficient function already known to this order in QCD. Our new result reduces the uncertainty due to the truncation of the perturbation series to a fraction of the uncertainty due to the present error of the strong coupling constant. We have also performed the corresponding but technically simpler computations for direct Higgs decay to bottom quarks and for the electromagnetic $R$-ratio in $e^{+} e^{-} \rightarrow$ hadrons, thus verifying important fifth-order results obtained only by one group so far.

KEYwords: Perturbative QCD, Higgs Physics

ARXIV EPRINT: 1707.01044 


\section{Contents}

1 Introduction 1

2 Theoretical framework and calculations 2

$\begin{array}{lll}3 & \text { Higgs decay to gluons } & 7\end{array}$

4 Higgs decay to bottom quarks $\quad 14$

5 The electromagnetic R-ratio $\quad 16$

$\begin{array}{llr}6 & \text { Summary } & 20\end{array}$

\section{Introduction}

The production and decay processes of the Higgs boson, discovered five years ago at CERN $[1,2]$ with a mass $M_{\mathrm{H}}$ of $125 \mathrm{GeV}$, are among the most important research topics in collider physics. The dominant standard-model decay is that to bottom quarks, $H \rightarrow \bar{b} b$ (+ hadrons). The QCD calculations of this decay mode have been completed up to the fourth order in the strong coupling $\alpha_{\mathrm{s}}$, see ref. [3] and references therein. A crucial component of this high accuracy is the next-to-next-to-next-to-next-to-leading order $\left(\mathrm{N}^{4} \mathrm{LO}\right)$ computation [4] of the decay to quarks via their direct coupling to the Higgs. This calculation, in which the quark mass can be neglected (except in the Yukawa coupling), has not been repeated so far.

The second important hadronic decay channel arises via $H \rightarrow g g$, where the coupling of the Higgs to gluons is predominantly mediated, in the standard model, by the top quark. Due to $M_{\mathrm{H}} \ll 2 m_{t}$, high-order QCD corrections to this process can be evaluated in an effective theory in which the top quark has been integrated out [5]; for $1 / m_{t}$ corrections up to NNLO see refs. [6, 7]. The resulting coefficient function for the effective Higgs coupling to gluons is known to $\mathrm{N}^{4} \mathrm{LO}$ [8-13]. The absorptive part of the corresponding vacuum polarization is not yet known at this order. The $\mathrm{N}^{3} \mathrm{LO}$ corrections have been computed in ref. [14] and checked in ref. [15] (except for their kinematic $\pi^{2}$ terms) and very recently in ref. [16]; see refs. $[5,17,18]$ for the previous orders.

In this article we present this hitherto missing fourth-order correction, thus completing the $\mathrm{N}^{4} \mathrm{LO}$ corrections to Higgs decay into hadrons in the limit of a heavy top quark and any number of massless flavours. We have also performed the computationally far simpler $\mathrm{N}^{4} \mathrm{LO}$ determination of the $H \rightarrow \bar{b} b$ decay rate and verified the result of ref. [4]. A somewhat more demanding but closely related computation is that of the $\mathrm{N}^{4} \mathrm{LO}$ corrections to the electromagnetic $R$-ratio for the process $e^{+} e^{-} \rightarrow$ hadrons. So far these corrections 
were determined only by one group [19-22]. We have also re-calculated this quantity to the fourth order, and find complete agreement for both the non-singlet and singlet contributions.

Our $\mathrm{N}^{4} \mathrm{LO}$ computations employ the same overall strategy as those by the KarlsruheMoscow group mentioned above (see also ref. [23]): the pole terms of the relevant correlation function are calculated in dimensional regularization [24, 25] at five loops, and subsequently the absorptive part is extracted. Our use of this approach has been made possible by the development of (a) ForCER [26-28], a FORM [29-31] program for the parametric reduction of four-loop self-energy integrals, and (b) a program [32] efficiently implementing the $R^{*}$ operation, see refs. [33-37], locally for the evaluation of $L$-loop pole terms in terms of $(L-1)$-loop integrals. In order to cope with the computations for $H \rightarrow g g$, which are far more demanding than those required to determine the five-loop beta function $[38,39]$, the latter program has undergone substantial modifications and extensions.

The remainder of this article is organized as follows: in section 2 we define our notations and briefly address some computational details. Our new $\mathrm{N}^{4} \mathrm{LO}$ result for the $H \rightarrow g g$ decay width is presented and discussed in section 3. Due to the rather large higher-order coefficients in the expansion in $\alpha_{\mathrm{s}}$, it is interesting to compare the results in the standard $\overline{\mathrm{MS}}$ scheme $[40,41]$ to those in a fairly common (and, in certain contexts, more physical) alternative, the miniMOM scheme [42, 43]. The transformation to this scheme, in contrast to other MOM schemes, is known to $\mathrm{N}^{4} \mathrm{LO}$ [44]; it has argued to be preferable to $\overline{\mathrm{MS}}$ for $H \rightarrow g g$ in a recent $\mathrm{N}^{3} \mathrm{LO}$ study [45].

In section 4 we briefly address the decay $H \rightarrow \bar{b} b$. We present the $\mathrm{N}^{4} \mathrm{LO}$ correction for a general gauge group and a general renormalization scale which has not been written down in the literature so far. The $\mathrm{N}^{4} \mathrm{LO}$ results in $\mathrm{QCD}$, which show a far less problematic behaviour at the only relevant case of $\alpha_{\mathrm{s}} \approx 0.1$ than their $H \rightarrow g g$ counterparts, have been known and discussed for more than ten years. Hence there is no need to go into more detail in this case. This is somewhat different for the $R$-ratio addressed in section 5 , despite its even smaller coefficients in the expansion in $\alpha_{\mathrm{s}}$, since this quantity is of physical relevance down to rather low scales and correspondingly high valus of $\alpha_{\mathrm{s}}$. Hence the size and scale (in-) stabilily of this quantity is illustrated in both the $\overline{\mathrm{MS}}$ and the miniMOM scheme at two low-scale reference points. We briefly summarize our results in section 6 .

\section{Theoretical framework and calculations}

Inclusive Higgs-boson decay to gluons. In the limit of a large top-quark mass and $n_{f}$ effectively massless flavours, the decay of the Higgs boson to hadrons can be calculated using the effective Lagrangian $[5,18]$

$$
\mathcal{L}_{\text {eff }}=\mathcal{L}_{\mathrm{QCD}\left(n_{f}\right)}-2^{1 / 4} G_{\mathrm{F}}^{1 / 2} C_{1} H G_{a}^{\mu \nu} G_{\mu \nu}^{a} .
$$

Here $H$ is the Higgs field, and $G_{a}^{\mu \nu}$ the renormalized gluon field-strength tensor for QCD with $n_{f}$ flavours and the Lagrangian $\mathcal{L}_{\mathrm{QCD}\left(n_{f}\right)}$. The renormalized coefficient function $C_{1}$ includes the top-mass dependence. $G_{\mathrm{F}} \simeq 1.1664 \cdot 10^{-5} \mathrm{GeV}^{-2}$ denotes the Fermi constant. 
At the leading order (LO) of perturbative QCD, eq. (2.1) implies that the Higgs decays to hadrons only via $H \rightarrow g g$. At the (next-to- $)^{n}$-leading order, $\mathrm{N}^{n} \mathrm{LO}$, up to $n$ additional partons occur in the final state. As usual, we will refer to the inclusive decay induced by eq. (2.1) as $H \rightarrow g g$ also beyond LO. The corresponding partial decay width $\Gamma_{H \rightarrow g g}$ can be related, via the optical theorem, to the imaginary part of the Higgs-boson self energy:

$$
\Gamma_{H \rightarrow g g}=\frac{\sqrt{2} G_{\mathrm{F}}}{M_{\mathrm{H}}}\left|C_{1}\right|^{2} \operatorname{Im} \Pi^{G G}\left(-M_{\mathrm{H}}^{2}-i \delta\right),
$$

where $M_{\mathrm{H}}$ is the Higgs boson mass, $\delta$ is an infinitesimally small positive real parameter and $\Pi^{G G}$ denotes the contribution to the self energy of the Higgs boson which is induced by its effective gluonic couplings as produced by eq. (2.1).

The Wilson coefficient $C_{1}$ can be extracted via a low-energy theorem from a decoupling relation [9] which relates the value of the strong coupling in a theory with $n_{f}$ light flavours,

$$
\alpha_{\mathrm{S}}\left(\mu^{2}\right) \equiv \alpha_{\mathrm{S}}^{\left(n_{f}\right)}\left(\mu^{2}\right)
$$

to its value $\alpha_{\mathrm{s}}^{\left(n_{f}+1\right)}$ in the corresponding theory with $n_{f}$ light flavours and one heavy flavour. The analytic QCD expression for $C_{1}$ up to $\mathrm{N}^{4} \mathrm{LO}$ has been provided in ref. [13] as a function of $\alpha_{\mathrm{S}}^{\left(n_{f}+1\right)}$ at the renormalization scale $\mu=\mu_{t}$, where $\mu_{t}=m_{t}\left(\mu_{t}\right)$ is the scale invariant (SI) top quark mass, i.e., the $\overline{\mathrm{MS}}$ mass evaluated at scale $\mu_{t}$. Using the decoupling relation [10-13], the renormalization group and the three-loop relation between the $\overline{\mathrm{MS}}$ mass and the on-shell (OS) mass [46, 47], we have rewritten the four-loop Wilson coefficient as a function of $\alpha_{\mathrm{s}}^{\left(n_{f}\right)}\left(\mu^{2}\right)$ at an arbitrary renormalization scale $\mu$ for the SI, $\overline{\mathrm{MS}}$ and OS top quark masses. The same has recently been done to three loops for the OS scheme in ref. [3].

For the convenience of the reader we include the resulting analytic expressions for $C_{1}$. These are presented in the form

$$
C_{1, \mathrm{X}}=-\frac{1}{3} a_{\mathrm{s}}\left(1+\sum_{n=1} c_{n, \mathrm{X}} a_{\mathrm{s}}^{n}\left(\mu^{2}\right)\right) \quad \text { with } \quad a_{\mathrm{s}} \equiv \frac{\alpha_{\mathrm{s}}}{4 \pi} .
$$

Here X labels the mass scheme employed, and we have indicated the reduced coupling $a_{\mathrm{s}}$ that we employ for all analytic expressions. The first two coefficients are the same in the above top-mass schemes up to the different definitions of masses entering $L_{t}=\ln \left(\mu^{2} / m_{t}^{2}\right)$,

$$
c_{1}=11, \quad c_{2}=\frac{2777}{18}+19 L_{t}-n_{f}\left[\frac{67}{6}-\frac{16}{3} L_{t}\right] .
$$

The $\mathrm{N}^{3} \mathrm{LO}$ and $\mathrm{N}^{4} \mathrm{LO}$ coefficients in the SI scheme read

$$
\begin{aligned}
c_{3, \mathrm{SI}}= & -\frac{2892659}{648}+\frac{897943}{144} \zeta_{3}+\frac{4834}{9} L_{t}+209 L_{t}^{2} \\
& +n_{f}\left[\frac{40291}{324}-\frac{110779}{216} \zeta_{3}+\frac{2912}{27} L_{t}+46 L_{t}^{2}\right] \\
& -n_{f}^{2}\left[\frac{6865}{486}-\frac{77}{27} L_{t}+\frac{32}{9} L_{t}^{2}\right]
\end{aligned}
$$




$$
\begin{aligned}
c_{4, \mathrm{SI}}= & -\frac{854201072999}{2041200}+\frac{28121193841}{75600} \zeta_{3}+\frac{4674213853}{28350} \zeta_{2}^{2}+\frac{913471669}{3780} \zeta_{5} \\
& -\frac{57744954}{4725} \ln 2 \zeta_{2}^{2}+\frac{93970579}{567} \ln ^{2} 2 \zeta_{2}-\frac{84531544}{2835} \ln ^{3} 2 \zeta_{2} \\
& -\frac{93970579}{3402} \ln ^{4} 2+\frac{42265772}{14175} \ln ^{5} 2-\frac{375882316}{567} a_{4}-\frac{338126176}{945} a_{5} \\
& -\frac{47987641}{216} L_{t}+\frac{9364157}{48} \zeta_{3} L_{t}+\frac{29494}{3} L_{t}^{2}+2299 L_{t}^{3} \\
& +n_{f}\left[\frac{76094378783}{2041200}-\frac{12171659669}{151200} \zeta_{3}+\frac{608462731}{113400} \zeta_{2}^{2}-\frac{22104149}{1890} \zeta_{5}\right. \\
& +\frac{37273868}{4725} \ln 2 \zeta_{2}^{2}-\frac{11679301}{1134} \ln ^{2} 2 \zeta_{2}+\frac{5453648}{2835} \ln ^{3} 2 \zeta_{2} \\
& +\frac{11679301}{6804} \ln { }^{4} 2-\frac{2726824}{14175} \ln ^{5} 2+\frac{23358602}{567} a_{4}+\frac{21814592}{945} a_{5} \\
& \left.+\frac{5343385}{162} L_{t}-\frac{258056}{9} \zeta_{3} L_{t}+\frac{12547}{9} L_{t}^{2}+\frac{1100}{3} L_{t}^{3}\right] \\
& +n_{f}^{2}\left[-\frac{48073}{108}+\frac{4091305}{1296} \zeta_{3}-\frac{576757}{540} \zeta_{2}^{2}-\frac{230}{3} \zeta_{5}-\frac{685}{27} \ln ^{2} 2 \zeta_{2}\right. \\
& \left.+\frac{685}{162} \ln ^{4} 2+\frac{2740}{27} a_{4}-\frac{42302}{27} L_{t}+\frac{28297}{36} \zeta_{3} L_{t}-\frac{5107}{54} L_{t}^{2}-\frac{628}{9} L_{t}^{3}\right] \\
& +n_{f}^{3}\left[-\frac{270407}{5832}+\frac{844}{27} \zeta_{3}+\frac{1924}{81} L_{t}-\frac{77}{27} L_{t}^{2}+\frac{64}{27} L_{t}^{3}\right]
\end{aligned}
$$

where $\zeta_{n}$ denotes the values of the Riemann $\zeta$-function and $a_{n}=\operatorname{Li}_{n}\left(\frac{1}{2}\right)=\sum_{k=1}^{\infty}\left(2^{k} k^{n}\right)^{-1}$. The corresponding expressions for the $\overline{\mathrm{MS}}$ and OS masses are given by

$$
\begin{aligned}
c_{3, \overline{\mathrm{MS}}}= & c_{3, \mathrm{SI}}-152 L_{t}-n_{f} \frac{128}{3} L_{t} \\
c_{4, \overline{\mathrm{MS}}}= & c_{4, \mathrm{SI}}-\frac{50186}{9} L_{t}-\frac{12692}{3} L_{t}^{2}-n_{f}\left[\frac{31282}{27} L_{t}+\frac{8408}{9} L_{t}^{2}\right] \\
& -n_{f}^{2}\left[\frac{136}{27} L_{t}-\frac{640}{9} L_{t}^{2}\right]
\end{aligned}
$$

and

$$
\begin{aligned}
c_{3, \mathrm{OS}}= & c_{3, \mathrm{SI}}+\frac{608}{3}+n_{f} \frac{512}{9}, \\
c_{4, \mathrm{OS}}= & c_{4, \mathrm{SI}}+\frac{297587}{27}+1216 \zeta_{2}-\frac{304}{3} \zeta_{3}+\frac{1216}{3} \ln 2 \zeta_{2}+6688 L_{t} \\
& +n_{f}\left[\frac{189238}{81}+\frac{416}{3} \zeta_{2}-\frac{256}{9} \zeta_{3}+\frac{1024}{9} \ln 2 \zeta_{2}+1472 L_{t}\right] \\
& -n_{f}^{2}\left[\frac{4352}{81}+\frac{512}{9} \zeta_{2}+\frac{1024}{9} L_{t}\right] .
\end{aligned}
$$

Our first calculation of the second component of eq. (2.2), $\operatorname{Im} \Pi^{G G}$, to $\mathrm{N}^{4} \mathrm{LO}$ is addressed below; for a typical Feynman diagram see the left part of figure 1 . The results are presented and combined with $C_{1}$ to $\mathrm{N}^{4} \mathrm{LO}$ results for $\Gamma_{H \rightarrow g g}$ in section 3. 

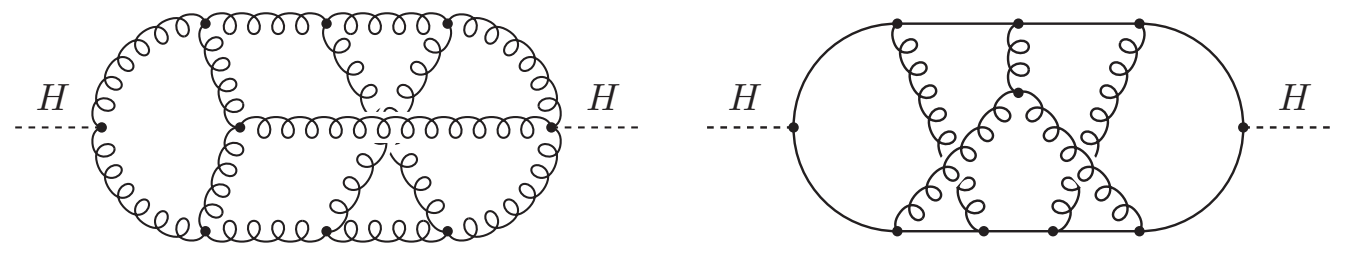

Figure 1. 5-loop Feynman diagrams evaluated for the $H \rightarrow g g$ and $H \rightarrow \bar{b} b$ decay rates.

Higgs decay to bottom quarks and the $\boldsymbol{R}$-ratio. As ref. [4], we compute the inclusive Higgs decay to bottom quarks at $\mathrm{N}^{4} \mathrm{LO}$ in the limit of a small bottom mass, keeping only the leading term proportional to the Yukawa coupling. The corresponding partial decay width can be extracted, again via the optical theorem, from the imaginary part of the bottom-Yukawa induced Higgs-boson self energy $\Pi^{B B}$,

$$
\Gamma_{H \rightarrow \bar{b} b}=\frac{G_{\mathrm{F}} M_{\mathrm{H}} m_{b}^{2}}{4 \sqrt{2} \pi} \tilde{R}\left(M_{\mathrm{H}}^{2}\right) \quad \text { with } \quad \tilde{R}(s)=\frac{\operatorname{Im} \Pi^{B B}(-s-i \delta)}{2 \pi s} .
$$

A diagram contributing to this process is shown in the right part of figure 1 .

The third observable we consider is the hadronic R-ratio, see refs. [19-22] and references therein, defined as

$$
R(s)=\frac{\sigma_{e^{+} e^{-} \rightarrow \text { hadrons }}}{\sigma_{e^{+} e^{-} \rightarrow \mu^{+} \mu^{-}}} .
$$

Away from the $Z$-pole, the most important contribution to $R(s)$ is given by the partial decay width of an off-shell photon into massless quarks. Here we re-compute the $\mathrm{N}^{4} \mathrm{LO}$ QCD corrections to this electromagnetic contribution. Analogous to the Higgs decay, this quantity can be extracted from the imaginary part of the photon self energy

$$
\Pi^{\mu \nu}\left(q^{2}\right)=\left(-g^{\mu \nu} q^{2}+q^{\mu} q^{\nu}\right) \Pi\left(q^{2}\right)
$$

via

$$
R^{\text {e.m. }}(s)=12 \pi \operatorname{Im} \Pi(-s-i \delta)=N_{R}\left[\left(\sum_{f} e_{f}^{2}\right) r(s)+\left(\sum_{f} e_{f}\right)^{2} r_{\mathrm{S}}(s)\right]
$$

with $N_{R}=3$ in QCD. The sum runs over $n_{f}$ quark flavours $f$ with electromagnetic charges $e_{f}$. The functions $r(s)$ and $r_{\mathrm{S}}(s)$ represent the respective non-singlet and singlet contributions to the $R$-ratio. Example diagrams for these two contributions are shown in figure 2.

Calculations. For all three observables under consideration, we are interested in the imaginary parts of self energies. These can be readily obtained by analytic continuation,

$$
\operatorname{Im} \Pi\left(-q^{2}-i \delta\right)=\operatorname{Im} e^{i \pi \varepsilon L} \Pi\left(q^{2}\right)=\sin (L \pi \varepsilon) \Pi\left(q^{2}\right),
$$

where $\varepsilon=\frac{1}{2}(4-D)$ is the dimensional regulator and $L$ the number of loops. The crucial point is now that the imaginary part of the self energy is suppressed by a factor of $\varepsilon$ :

$$
\sin (L \pi \varepsilon)=L \pi \varepsilon\left(1-\frac{1}{3 !}(L \pi \varepsilon)^{2}+\frac{1}{5 !}(L \pi \varepsilon)^{4}+\ldots\right) .
$$

Consequently the finite part of $\operatorname{Im} \Pi\left(-q^{2}\right)$ can be obtained from the $1 / \varepsilon$ term of $\Pi\left(q^{2}\right)$. 

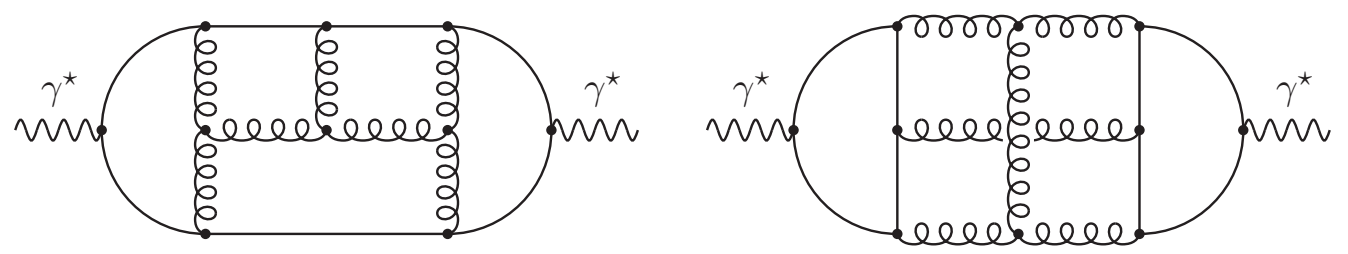

Figure 2. Sample non-singlet (left) and singlet (right) Feynman diagrams for which the $1 / \varepsilon$ pole terms were computed in our re-calculation of the electromagnetic $R$-ratio at $\mathrm{N}^{4} \mathrm{LO}$.

To compute the single poles we employ the $R^{*}$-operation for Feynman diagrams with arbitrary numerators [32] to express the poles of five-loop diagrams in terms of four-loop diagrams. The $R^{*}$-operation thus allows us to compute all ingredients required here using the FORCER program [26-28], which automates the reduction and calculation of massless four-loop self energy diagrams. The same approach was used in ref. [39] to compute the five-loop beta function for an arbitrary simple compact gauge group.

However, the Higgs decay to gluons poses a much greater computational challenge: the diagrams are all quartically divergent. In order to infrared rearrange the diagrams, the superficial degree of divergence of the diagrams must be logarithmic. We achieve this by computing the fourth order coefficient of the Taylor expansion in the external momentum $q$ about the point $q=0$, i.e. we apply the differential operator

$$
\left.\frac{1}{4 !} q^{\mu_{1}} q^{\mu_{2}} q^{\mu_{3}} q^{\mu_{4}} \frac{\partial}{\partial q^{\mu_{1}}} \frac{\partial}{\partial q^{\mu_{2}}} \frac{\partial}{\partial q^{\mu_{3}}} \frac{\partial}{\partial q^{\mu_{4}}}(\bullet \quad)\right|_{q=0}
$$

to all Feynman diagrams. As a result, an 'explosion' of terms, with complicated numerator structures, is created. To deal with this complexity, we have significantly improved our algorithms, in particular for the reduction of high rank tensor vacuum graphs.

The Feynman diagrams for all three cases have been generated using QGRAF [48] and were then processed by a Form [29-31] program that assigns the topology and determines the colour factor using the program of ref. [49]. Diagrams of the same topology, colour factor, and maximal power of $n_{\ell}$ have been combined to meta diagrams for computational efficiency. Lower-order self-energy insertions have been treated as described in ref. [50].

In the case of $\Pi^{G G}$, this procedure leads to 1 one-loop, 5 two-loop, 38 three-loop, 394 four-loop and 6405 five-loop meta diagrams. These are fewer meta diagrams than for our calculation of the 5-loop beta function using the background-field method (by a factor 0.68 to 0.69 beyond two loops), but the present diagrams are much harder, as discussed above. The computations were performed on the same set of a modern and somewhat dated machines as used for our five-loop beta function [39], and required an order of magnitude more time.

In the much more modest cases of $\Pi^{B B}$ and $\Pi$ in eqs. (2.12) and (2.15), for which we can use the same diagram set which different external vertices and projections, we computed 1 one-loop, 2 two-loop, 9 three-loop, 64 four-loop and 804 five-loop meta diagrams.

We have checked our results by computing all diagrams by at least two different infrared rearrangements. A different rearrangement results in the computation of a different set of 
counterterms, but should give the same result in the end. This therefore constitutes a highly non-trivial consistency check of our setup.

The first strategy of IR rearrangement consists of attaching external momenta around the line with the worst IR divergence, for example
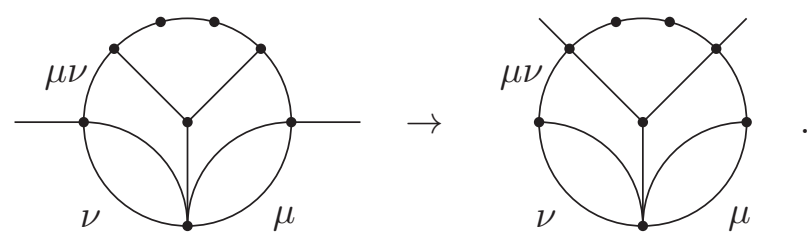

The resulting integral is an $L$-loop 'carpet' integral, which can be reduced to a $L-1$ loop propagator integral [28]. By attaching the external momenta around the worst IR divergent line, the number of counterterms that include this line is limited.

The second IR rearrangement consists of inserting a mass into the worst IR divergent propagator:
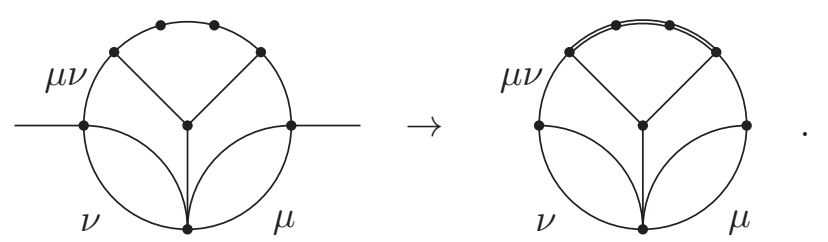

The resulting counterterm diagrams can always be split up into a massive one-loop vacuum bubble and an $L-1$ loop massless propagator integral which can be computed using FORCER. The advantage of this method is that the massive line cannot be part of any IR counterterm and that the 'carpet' rule reduction is avoided. Overall, this rearrangement is about $20 \%$ to $50 \%$ faster than attaching external momenta.

\section{$3 \quad$ Higgs decay to gluons}

After the calculation of the Feynman diagrams, the extraction of the absorptive part and its renormalization, the coefficients $g_{n}$ up to $\mathrm{N}^{4} \mathrm{LO}$ in

$$
\frac{4 \pi}{N_{A} q^{4}} \operatorname{Im} \Pi^{G G}\left(q^{2}\right) \equiv G\left(q^{2}\right)=1+\sum_{n=1} g_{n} a_{\mathrm{s}}^{n},
$$

with $N_{A}=8$ in QCD, are found to be

$$
\begin{aligned}
g_{1}= & \frac{73}{3} C_{A}-\frac{14}{3} n_{f} \\
g_{2}= & C_{A}^{2}\left[\frac{37631}{54}-\frac{242}{3} \zeta_{2}-110 \zeta_{3}\right]-C_{A} n_{f}\left[\frac{6665}{27}-\frac{88}{3} \zeta_{2}+4 \zeta_{3}\right] \\
& -C_{F} n_{f}\left[\frac{131}{3}-24 \zeta_{3}\right]+n_{f}^{2}\left[\frac{508}{27}-\frac{8}{3} \zeta_{2}\right]
\end{aligned}
$$




$$
\begin{aligned}
g_{3}= & C_{A}^{3}\left[\frac{15420961}{729}-\frac{45056}{9} \zeta_{2}-\frac{178156}{27} \zeta_{3}+\frac{3080}{3} \zeta_{5}\right] \\
& -C_{A}^{2} n_{f}\left[\frac{2670508}{243}-\frac{8084}{3} \zeta_{2}-\frac{9772}{9} \zeta_{3}+\frac{80}{3} \zeta_{5}\right] \\
& -C_{F} C_{A} n_{f}\left[\frac{23221}{9}-\frac{572}{3} \zeta_{2}-1364 \zeta_{3}-160 \zeta_{5}\right] \\
& +C_{F}^{2} n_{f}\left[\frac{221}{3}+192 \zeta_{3}-320 \zeta_{5}\right]+C_{A} n_{f}^{2}\left[\frac{413308}{243}-\frac{1384}{3} \zeta_{2}+\frac{56}{9} \zeta_{3}\right] \\
& +C_{F} n_{f}^{2}\left[440-\frac{104}{3} \zeta_{2}-240 \zeta_{3}\right]-n_{f}^{3}\left[\frac{57016}{729}-\frac{224}{9} \zeta_{2}-\frac{64}{27} \zeta_{3}\right]
\end{aligned}
$$

and

$$
\begin{aligned}
& g_{4}=C_{A}^{4}\left[\frac{5974862279}{8748}-\frac{58922654}{243} \zeta_{2}-\frac{25166402}{81} \zeta_{3}+\frac{292556}{45} \zeta_{2}^{2}+\frac{266200}{9} \zeta_{2} \zeta_{3}\right. \\
& \left.+\frac{1817200}{27} \zeta_{5}+\frac{121000}{9} \zeta_{3}^{2}-\frac{96250}{9} \zeta_{7}\right] \\
& -\frac{d_{A}^{a b c d} d_{A}^{a b c d}}{N_{A}}\left[\frac{6416}{27}-\frac{54160}{9} \zeta_{3}-\frac{1408}{5} \zeta_{2}^{2}+\frac{13760}{3} \zeta_{5}-\frac{19360}{3} \zeta_{3}^{2}+\frac{6160}{3} \zeta_{7}\right] \\
& -C_{A}^{3} n_{f}\left[\frac{1025827736}{2187}-\frac{41587004}{243} \zeta_{2}-\frac{8812352}{81} \zeta_{3}+\frac{211736}{45} \zeta_{2}^{2}+9680 \zeta_{2} \zeta_{3}\right. \\
& \left.+\frac{109220}{9} \zeta_{5}-\frac{8800}{9} \zeta_{3}^{2}+\frac{3500}{9} \zeta_{7}\right] \\
& -C_{A}^{2} C_{F} n_{f}\left[\frac{348948545}{2916}-22340 \zeta_{2}-\frac{1869710}{27} \zeta_{3}+\frac{656}{15} \zeta_{2}^{2}+\frac{19360}{3} \zeta_{2} \zeta_{3}\right. \\
& \left.-\frac{35540}{3} \zeta_{5}+\frac{17600}{3} \zeta_{3}^{2}-\frac{7000}{3} \zeta_{7}\right] \\
& +C_{A} C_{F}^{2} n_{f}\left[\frac{609521}{162}-\frac{484}{3} \zeta_{2}+\frac{450374}{27} \zeta_{3}+\frac{352}{15} \zeta_{2}^{2}-\frac{63040}{3} \zeta_{5}-5600 \zeta_{7}\right] \\
& +C_{F}^{3} n_{f}\left[\frac{1034}{3}-388 \zeta_{3}-4560 \zeta_{5}+5600 \zeta_{7}\right] \\
& +\frac{d_{F}^{a b c d} d_{A}^{a b c d}}{N_{A}} n_{f}\left[\frac{44864}{27}-\frac{140128}{9} \zeta_{3}-\frac{3328}{5} \zeta_{2}^{2}+\frac{20800}{3} \zeta_{5}-\frac{14080}{3} \zeta_{3}^{2}+\frac{2240}{3} \zeta_{7}\right] \\
& +C_{A}^{2} n_{f}^{2}\left[\frac{26855351}{243}-\frac{3479386}{81} \zeta_{2}-\frac{83536}{9} \zeta_{3}+\frac{19472}{15} \zeta_{2}^{2}+\frac{1760}{3} \zeta_{2} \zeta_{3}-\frac{1240}{9} \zeta_{5}+\frac{160}{9} \zeta_{3}^{2}\right] \\
& +C_{F} C_{A} n_{f}^{2}\left[\frac{29816212}{729}-\frac{71888}{9} \zeta_{2}-\frac{563948}{27} \zeta_{3}+\frac{224}{15} \zeta_{2}^{2}+\frac{7040}{3} \zeta_{2} \zeta_{3}-\frac{7000}{3} \zeta_{5}-\frac{640}{3} \zeta_{3}^{2}\right] \\
& +C_{F}^{2} n_{f}^{2}\left[\frac{90491}{81}-\frac{200}{3} \zeta_{2}-\frac{138968}{27} \zeta_{3}-\frac{352}{15} \zeta_{2}^{2}+4400 \zeta_{5}+640 \zeta_{3}^{2}\right]
\end{aligned}
$$




$$
\begin{aligned}
& -\frac{d_{F}^{a b c d} d_{F}^{a b c d}}{N_{A}} n_{f}^{2}\left[\frac{68096}{27}-\frac{39424}{9} \zeta_{3}-\frac{1024}{5} \zeta_{2}^{2}+1280 \zeta_{5}-\frac{2560}{3} \zeta_{3}^{2}\right] \\
& -C_{A} n_{f}^{3}\left[\frac{46491973}{4374}-\frac{1099028}{243} \zeta_{2}-\frac{23720}{81} \zeta_{3}+\frac{1408}{9} \zeta_{2}^{2}-\frac{320}{9} \zeta_{2} \zeta_{3}-\frac{800}{27} \zeta_{5}\right] \\
& -C_{F} n_{f}^{3}\left[\frac{2282351}{729}-\frac{6224}{9} \zeta_{2}-\frac{5200}{3} \zeta_{3}+\frac{640}{3} \zeta_{2} \zeta_{3}\right] \\
& +n_{f}^{4}\left[\frac{773024}{2187}-\frac{40640}{243} \zeta_{2}-\frac{2240}{81} \zeta_{3}+\frac{64}{9} \zeta_{2}^{2}\right] .
\end{aligned}
$$

Eqs. (3.2)-(3.4) agree with the previous results in refs. [5, 14-18] ( $n_{\ell}$ instead of $n_{f}$ is often used for the number of light flavours) eq. (3.5) represents the main new result of the present article. In all these equations $T_{F}=1 / 2$ has been inserted; this factor can be re-instated by substituting $n_{f} \rightarrow 2 T_{F} n_{f}$ in all terms that do not involve quartic group invariants.

The coefficients (3.2)-(3.5) are valid for the standard choice $\mu^{2}=q^{2}$ of the renormalization scale. The additional terms for $\mu^{2} \neq q^{2}$ can be obtained from the scale invariance of $\left(\beta\left(a_{\mathrm{s}}\right) / a_{\mathrm{s}}\right)^{2} \operatorname{Im} \Pi^{G G}\left(q^{2}\right)[5,14]$. This can be done, e.g., by inserting the expansion of $a_{\mathrm{S}}\left(q^{2}\right)$ in terms of $a_{\mathrm{s}}\left(\mu^{2}\right)$ which can be read off to the order required here, for example, from eq. (2.9) and footnote 2 of ref. [51]. The resulting generalizations of eqs. (3.2)-(3.5) read

$$
\begin{aligned}
g_{1}\left(L_{q}\right)=g_{1} & -2 \beta_{0} L_{q} \\
g_{2}\left(L_{q}\right)=g_{2} & -\left(4 \beta_{1}+3 \beta_{0} g_{1}\right) L_{q}+3 \beta_{0}^{2} L_{q}^{2} \\
g_{3}\left(L_{q}\right)=g_{3} & -\left(6 \beta_{2}+5 \beta_{1} g_{1}+4 \beta_{0} g_{2}\right) L_{q}+\left(13 \beta_{0} \beta_{1}+6 \beta_{0}^{2} g_{1}\right) L_{q}^{2}-4 \beta_{0}^{3} L_{q}^{3}, \\
g_{4}\left(L_{q}\right)=g_{4} & -\left(8 \beta_{3}+7 \beta_{2} g_{1}+6 \beta_{1} g_{2}+5 \beta_{0} g_{3}\right) L_{q} \\
+ & \left(12 \beta_{1}^{2}+22 \beta_{0} \beta_{2}+43 / 2 \beta_{0} \beta_{1} g_{1}+10 \beta_{0}^{2} g_{2}\right) L_{q}^{2} \\
& -\left(83 / 3 \beta_{0}^{2} \beta_{1}+10 \beta_{0}^{3} g_{1}\right) L_{q}^{3}+5 \beta_{0}^{4} L_{q}^{4}
\end{aligned}
$$

in terms of the above coefficients $g_{n}$, the coefficients $\beta_{n}$ of the $\overline{\text { MS }}$ beta function up to $\mathrm{N}^{3} \mathrm{LO}[52,53]$ and $L_{q} \equiv \ln \left(q^{2} / \mu^{2}\right)$. The resulting explicit coefficients up to $g_{3}$ agree with eq. (26) of ref. [3], where the definitions of $L$ and $a_{\mathrm{s}}$ are slightly different.

At the scale $\mu^{2}=q^{2}$ the numerical expansion of the function $G\left(q^{2}\right)$ in eq. (3.1) is given by

$$
\begin{aligned}
& n_{f}=1: 1+5.4377939 \alpha_{\mathrm{s}}+20.720313 \alpha_{\mathrm{s}}^{2}+58.92184 \alpha_{\mathrm{s}}^{3}+118.0078 \alpha_{\mathrm{s}}^{4}+\ldots, \\
& n_{f}=3: 1+4.6950708 \alpha_{\mathrm{s}}+13.472440 \alpha_{\mathrm{s}}^{2}+20.66395 \alpha_{\mathrm{s}}^{3}-15.96239 \alpha_{\mathrm{s}}^{4}+\ldots, \\
& n_{f}=5: 1+3.9523478 \alpha_{\mathrm{s}}+6.9555141 \alpha_{\mathrm{s}}^{2}-6.851753 \alpha_{\mathrm{s}}^{3}-75.25914 \alpha_{\mathrm{s}}^{4}+\ldots, \\
& n_{f}=7: 1+3.2096247 \alpha_{\mathrm{s}}+1.1695355 \alpha_{\mathrm{s}}^{2}-24.45788 \alpha_{\mathrm{s}}^{3}-76.99773 \alpha_{\mathrm{s}}^{4}+\ldots, \\
& n_{f}=9: 1+2.4669016 \alpha_{\mathrm{s}}-3.8854956 \alpha_{\mathrm{s}}^{2}-32.98703 \alpha_{\mathrm{s}}^{3}-37.30247 \alpha_{\mathrm{s}}^{4}+\ldots
\end{aligned}
$$

for QCD with up to 5 quark families, i.e., $n_{f}=1, \ldots, 9$ light flavours. In the only physically relevant case of $n_{f}=5$ the effect of the fourth-order correction is larger than that of the previous order for $\alpha_{\mathrm{s}} \gtrsim 0.1$. It is clear from eqs. (3.7), though, that this is not 
a generic feature of the QCD perturbation series, but a consequence of the 'accidentally' small size of the third-order term for this number of flavours. A similar situation has been observed for Higgs decay to bottom quarks, see eq. (8) of ref. [4] and eq. (4.6) below.

The fourth-order coefficient $g_{4}$ in eq. (3.5) is the first to receive contributions from quartic group invariants. The overall effect of these terms is small in the range of $n_{f}$ considered above; nullifying all these terms changes the coefficients of $\alpha_{\mathrm{s}}^{4}$ in eqs. (3.7) by about $5 \%$ or less for $n_{f} \neq 3$. For $n_{f}=3$, the relative effect is larger since the coefficient is atypically small.

As discussed in ref. [14], the $\zeta_{2}=\pi^{2} / 6$ contributions in eqs. (3.3)-(3.4) only arise from the analytic continuation (2.16) and are predictable from lower-order results. The same holds for the terms linear in $\zeta_{2}$ in eq. (3.5). However, the 'genuine' five-loop contributions from the functions $\Pi^{G G}\left(q^{2}\right)$ include terms with $\zeta_{2}^{2}$, so not all powers of $\pi^{2}$ are 'kinematical' from this order onwards. The numerical decomposition of the expansion in eq. (3.1) into the 'genuine' and 'kinematical' contributions (underlined below) reads

$$
\begin{aligned}
G\left(q^{2}\right)= & 1+3.952348 \alpha_{\mathrm{s}}+(10.629125-\underline{3.673611}) \alpha_{\mathrm{s}}^{2} \\
& +(28.57606-\underline{35.42782}) \alpha_{\mathrm{s}}^{3}+(89.55798-\underline{164.81711}) \alpha_{\mathrm{s}}^{4}
\end{aligned}
$$

for the physical case of $n_{f}=5$. The numbers up to order $\alpha_{\mathrm{s}}^{3}$ agree, of course, with ref. [14]; the $n_{f}$-dependent decomposition of our new coefficient $g_{4}$ in eq. (3.5) is

$$
\begin{aligned}
g_{4}= & 1267.05129-\underline{1048.43622}-(394.681626-\underline{281.704409}) n_{f} \\
& +(37.9589880-\underline{25.1937144}) n_{f}^{2}-(1.28868582-\underline{0.89082162}) n_{f}^{3} \\
& +(0.01284135-\underline{0.01026045}) n_{f}^{4} .
\end{aligned}
$$

The cancellations between the genuine and kinematic contributions are somewhat less striking than for the corresponding contribution to $H \rightarrow \bar{b} b$, see eq. (7) of ref. [4], yet the conclusion remains the same: it is not possible to obtain reliable results without computing the genuine contributions.

The decay rate $\Gamma_{H \rightarrow g g}$ in the limit of a heavy top quark and $n_{f}$ effectively massless flavours is obtained by combining eqs. (3.1)-(3.6) with the corresponding expansion of the coefficient function $C_{1}$ in eqs. (2.4)-(2.11) above. The resulting $K$-factors, defined by $\Gamma=K \Gamma_{\text {Born }}$ at $\mu^{2}=M_{\mathrm{H}}^{2}$, see eq. (3.12) below, are given by

$$
\begin{aligned}
& K_{\mathrm{SI}}\left(n_{f}=1\right)=1+7.188498 \alpha_{\mathrm{s}}+32.65167 \alpha_{\mathrm{s}}^{2}+112.015 \alpha_{\mathrm{s}}^{3}+298.873 \alpha_{\mathrm{s}}^{4}+\ldots, \\
& K_{\mathrm{SI}}\left(n_{f}=3\right)=1+6.445775 \alpha_{\mathrm{s}}+23.74728 \alpha_{\mathrm{s}}^{2}+56.0755 \alpha_{\mathrm{s}}^{3}+62.4363 \alpha_{\mathrm{s}}^{4}+\ldots, \\
& K_{\mathrm{SI}}\left(n_{f}=5\right)=1+5.703052 \alpha_{\mathrm{s}}+15.57384 \alpha_{\mathrm{s}}^{2}+12.5520 \alpha_{\mathrm{s}}^{3}-72.0916 \alpha_{\mathrm{s}}^{4}+\ldots \\
& K_{\mathrm{SI}}\left(n_{f}=7\right)=1+4.960329 \alpha_{\mathrm{s}}+8.131350 \alpha_{\mathrm{s}}^{2}-19.3879 \alpha_{\mathrm{s}}^{3}-123.853 \alpha_{\mathrm{s}}^{4}+\ldots, \\
& K_{\mathrm{SI}}\left(n_{f}=9\right)=1+4.217606 \alpha_{\mathrm{s}}+1.419805 \alpha_{\mathrm{s}}^{2}-40.5769 \alpha_{\mathrm{s}}^{3}-110.998 \alpha_{\mathrm{s}}^{4}+\ldots
\end{aligned}
$$

for a scale-invariant top mass $\mu_{t}=164 \mathrm{GeV}$, and by

$$
\begin{aligned}
& K_{\mathrm{OS}}\left(n_{f}=1\right)=1+7.188498 \alpha_{\mathrm{s}}+32.61874 \alpha_{\mathrm{s}}^{2}+112.031 \alpha_{\mathrm{s}}^{3}+300.278 \alpha_{\mathrm{s}}^{4}+\ldots, \\
& K_{\mathrm{OS}}\left(n_{f}=3\right)=1+6.445775 \alpha_{\mathrm{s}}+23.69992 \alpha_{\mathrm{s}}^{2}+56.1329 \alpha_{\mathrm{s}}^{3}+64.5259 \alpha_{\mathrm{s}}^{4}+\ldots,
\end{aligned}
$$




$$
\begin{aligned}
& K_{\mathrm{OS}}\left(n_{f}=5\right)=1+5.703052 \alpha_{\mathrm{s}}+15.51204 \alpha_{\mathrm{s}}^{2}+12.6660 \alpha_{\mathrm{s}}^{3}-69.3287 \alpha_{\mathrm{s}}^{4}+\ldots \\
& K_{\mathrm{OS}}\left(n_{f}=7\right)=1+4.960329 \alpha_{\mathrm{s}}+8.055116 \alpha_{\mathrm{s}}^{2}-19.2021 \alpha_{\mathrm{s}}^{3}-120.458 \alpha_{\mathrm{s}}^{4}+\ldots \\
& K_{\mathrm{OS}}\left(n_{f}=9\right)=1+4.217606 \alpha_{\mathrm{s}}+1.329135 \alpha_{\mathrm{s}}^{2}-40.3039 \alpha_{\mathrm{s}}^{3}-107.042 \alpha_{\mathrm{s}}^{4}+\ldots
\end{aligned}
$$

for an on-shell top mass of $M_{t}=173 \mathrm{GeV}$. The effect of the coefficient functions is positive, except for their $\mathrm{N}^{3} \mathrm{LO}$ and $\mathrm{N}^{4} \mathrm{LO}$ contributions at large $n_{f}$.

The mass- and scale-dependent expansion coefficients $\gamma_{n}$ for the physical case $n_{f}=5$ in

$$
\Gamma_{H \rightarrow g g}=\frac{G_{F} M_{\mathrm{H}}^{3}}{36 \pi^{3} \sqrt{2}} \sum_{n=0} \gamma_{n}\left(M_{\mathrm{H}}, m_{t}, \mu\right)\left(\alpha_{\mathrm{s}}\left(\mu^{2}\right)\right)^{n+2}
$$

(the $n=0$ contribution is the Born result) are given by $\gamma_{0}=1$ and

$$
\begin{aligned}
\gamma_{1, \mathrm{SI}}= & 5.703052-1.220188 L_{H} \\
\gamma_{2, \mathrm{SI}}= & 15.887961-0.578375 L_{t H}-10.927911 L_{H}+1.116644 L_{H}^{2} \\
\gamma_{3, \mathrm{SI}}= & 14.59257-3.94891 L_{t H}+0.352863 L_{t H}^{2} \\
& -\left(43.14427-1.41145 L_{t H}\right) L_{H}+13.78227 L_{H}^{2}-0.908344 L_{H}^{3} \\
\gamma_{4, \mathrm{SI}}= & -66.75046-11.35498 L_{t H}+2.91649 L_{t H}^{2}-0.215280 L_{t H}^{3} \\
& -\left(62.02230-12.61251 L_{t H}+1.07640 L_{t H}^{2}\right) L_{H} \\
& +\left(71.32360-2.15280 L_{t H}\right) L_{H}^{2}-14.37869 L_{H}^{3}+0.692719 L_{H}^{4}
\end{aligned}
$$

with $L_{H}=\ln \left(M_{\mathrm{H}}^{2} / \mu^{2}\right)$ and $L_{t H}=\ln \left(\mu_{t}^{2} / M_{\mathrm{H}}^{2}\right)$ in terms of the scale-invariant top-quark mass. The corresponding OS-mass coefficient have the same form at NLO and NNLO, and read

$$
\begin{aligned}
& \gamma_{3, \mathrm{OS}}=\gamma_{3, \mathrm{SI}}+0.490940, \\
& \gamma_{4, \mathrm{OS}}=\gamma_{4, \mathrm{SI}}+4.21311-0.89856 L_{t H}-1.49760 L_{H},
\end{aligned}
$$

where the top-mass logarithms are now given by $L_{t H}=\ln \left(M_{t}^{2} / M_{\mathrm{H}}^{2}\right)$.

The size of the higher order corrections and the improvement of the renormalization scale dependence from NLO to $\mathrm{N}^{4} \mathrm{LO}$ is illustrated in figure 3 for $\left(\beta\left(a_{\mathrm{s}}\right) / a_{\mathrm{s}}\right)^{2} \operatorname{Im} \Pi^{G G}\left(M_{\mathrm{H}}^{2}\right)$, recall the discussion above eq. (3.6), and for $\Gamma_{H \rightarrow g g}$ in eq. (3.12). The first term in the expansion has been normalized for both quantities, i.e., $\Gamma_{0}$ in the figure is given by

$$
\Gamma_{0}=G_{F} M_{\mathrm{H}}^{3} /\left(36 \pi^{3} \sqrt{2}\right) \cdot\left(\alpha_{\mathrm{s}}\left(M_{\mathrm{H}}^{2}\right)\right)^{2} \quad \text { with } \quad \alpha_{\mathrm{s}}\left(M_{\mathrm{H}}^{2}\right)=0.11264
$$

which corresponds to $\alpha_{\mathrm{S}}\left(M_{\mathrm{Z}}^{2}\right)=0.118$. The normalized decay rate is shown for an SI mass of $164 \mathrm{GeV}$. The very similar results for an OS mass of $173 \mathrm{GeV}$ are shown below.

The effect of the $\mathrm{N}^{4} \mathrm{LO}$ correction to $\Gamma_{H \rightarrow g g}$ is $-0.6 \%$ at $\mu=M_{\mathrm{H}}$, and $-0.8 \% /+0.9 \%$ at $\mu=0.5 / 2 M_{\mathrm{H}}$, respectively. The total $\mathrm{N}^{4} \mathrm{LO}$ result at $\mu=M_{\mathrm{H}}$ is $1.846 \Gamma_{0}$, and its range in the above scale interval is $(1.836-1.848) \Gamma_{0}$. The $\mathrm{N}^{4} \mathrm{LO}$ scale variation between $\mu=1 / 3 M_{\mathrm{H}}$ and $\mu=3 M_{\mathrm{H}}$ is as small as $0.9 \%$ (full width), a reduction of almost a factor of four with respect to the corresponding $\mathrm{N}^{3} \mathrm{LO}$ result. The dependence of $\Gamma_{H \rightarrow g g}$ on the top mass is very weak: changing $\mu_{t}$ by $4 \mathrm{GeV}$ [54] changes the result by only $0.04 \%$. The largest uncertainty at $\mathrm{N}^{4} \mathrm{LO}$ is due to $\alpha_{\mathrm{s}}$ : changing $\alpha_{\mathrm{S}}\left(M_{\mathrm{Z}}^{2}\right)$ by $1 \%$ changes the result by $2.5 \%$. 

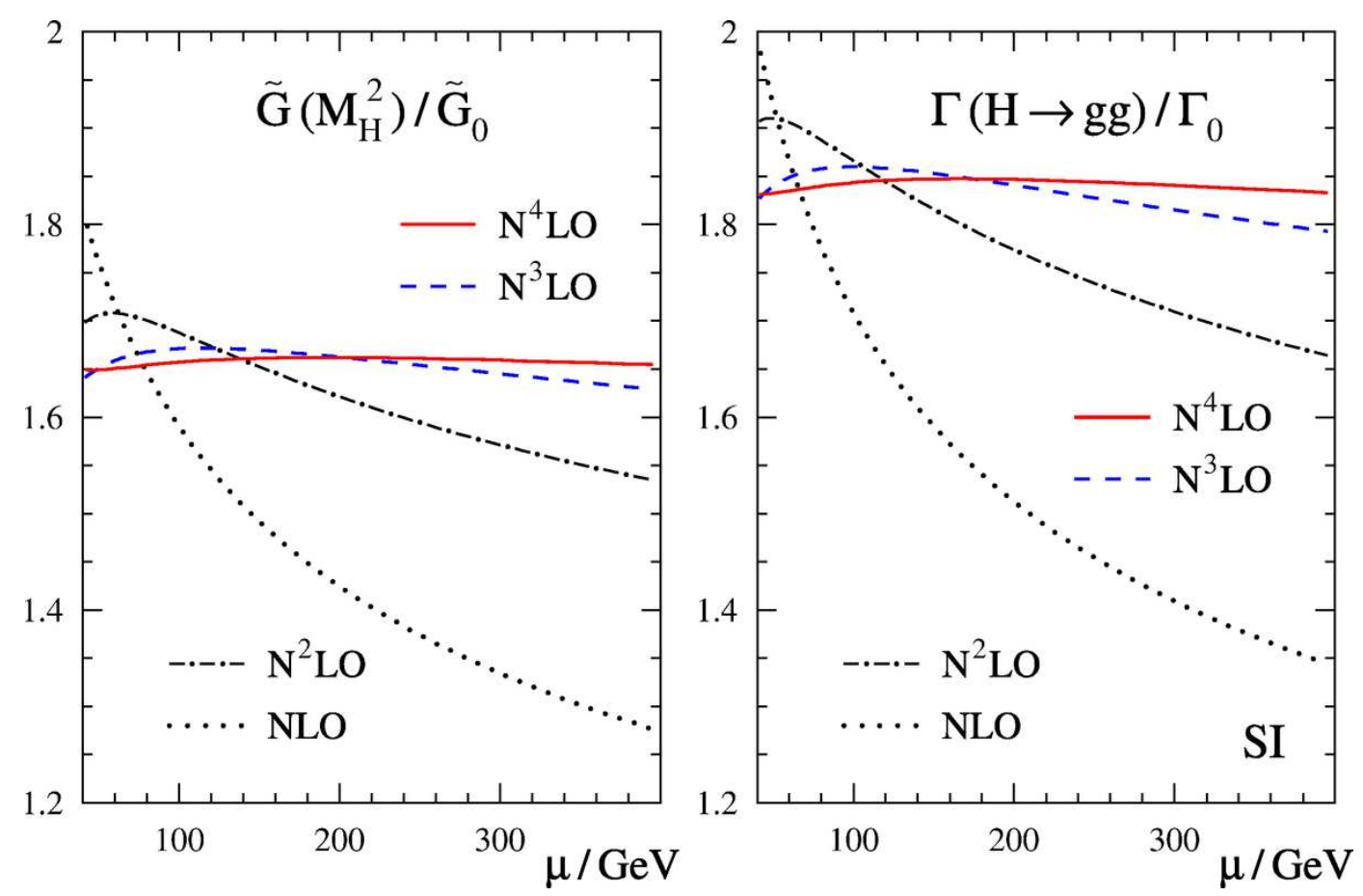

Figure 3. The renormalization-scale dependence of $\widetilde{G}=\left(\beta\left(a_{\mathrm{s}}\right) / a_{\mathrm{s}}\right)^{2} G\left(M_{\mathrm{H}}^{2}\right)$, with $G\left(q^{2}\right)$ defined in eq. (3.1), at $n_{f}=5$ (left panel), and of the decay width $\Gamma_{H \rightarrow g g}$ (right panel), both normalized as discussed in the text, up to $\mathrm{N}^{4} \mathrm{LO}$ in $\overline{\mathrm{MS}}$ for $\alpha_{\mathrm{s}}\left(M_{\mathrm{Z}}^{2}\right)=0.118, M_{\mathrm{H}}=125 \mathrm{GeV}$ and $\mu_{t}=164 \mathrm{GeV}$.

Averaging the result at $\mu=M_{\mathrm{H}}$ and the central value of the above scale interval, and using the shift at $\mu=M_{\mathrm{H}}$ from $\mathrm{N}^{3} \mathrm{LO}$ to $\mathrm{N}^{4} \mathrm{LO}$ (or twice the width of the above scale range) for a conservative estimate of the series expansion uncertainty, the $\mathrm{N}^{4} \mathrm{LO}$ result without $1 / m_{t}$ corrections and light-quark mass effects - can be summarized as

$$
\Gamma_{\mathrm{N}^{4} \mathrm{LO}}(H \rightarrow g g)=\Gamma_{0}\left(1.844 \pm 0.011_{\text {series }} \pm 0.045_{\alpha_{\mathrm{s}}\left(M_{\mathrm{Z}}\right), 1 \%}\right) .
$$

The uncertainty due to the truncation of the perturbation series at $\mathrm{N}^{4} \mathrm{LO}$ is definitely much smaller than the uncertainty due to that of $\alpha_{\mathrm{s}}\left(M_{\mathrm{Z}}\right)$ which may exceed the value of $1 \%$ quoted by the Particle Data Group [55]; see ref. [54] for a recent deviating analysis.

We conclude our discussion of $\Gamma_{H \rightarrow g g}$ by re-expressing its perturbative expansion in another renormalization scheme, the miniMOM scheme [42, 43]. The transformation from $\overline{\mathrm{MS}}$ to miniMOM and the beta function in this scheme have been derived at $\mathrm{N}^{4} \mathrm{LO}$ in ref. [44].

The decay width (3.12) in the OS scheme for the top mass can be readily transformed by expressing $\alpha_{\mathrm{s}}$ in terms of $\alpha_{\mathrm{s}, \mathrm{MM}}$. For the Landau-gauge miniMOM scheme one finds

$$
\begin{aligned}
& K_{\mathrm{OS}}^{\mathrm{MM}}\left(n_{f}=1\right)=1+5.123905 \alpha_{\mathrm{s}}+10.56499 \alpha_{\mathrm{s}}^{2}-7.47722 \alpha_{\mathrm{s}}^{3}-112.155 \alpha_{\mathrm{s}}^{4}+\ldots, \\
& K_{\mathrm{OS}}^{\mathrm{MM}}\left(n_{f}=3\right)=1+4.734860 \alpha_{\mathrm{s}}+7.406951 \alpha_{\mathrm{s}}^{2}-14.9763 \alpha_{\mathrm{s}}^{3}-91.2437 \alpha_{\mathrm{s}}^{4}+\ldots, \\
& K_{\mathrm{OS}}^{\mathrm{MM}}\left(n_{f}=5\right)=1+4.345814 \alpha_{\mathrm{s}}+4.379443 \alpha_{\mathrm{s}}^{2}-21.5506 \alpha_{\mathrm{s}}^{3}-71.9231 \alpha_{\mathrm{s}}^{4}+\ldots \\
& K_{\mathrm{OS}}^{\mathrm{MM}}\left(n_{f}=7\right)=1+3.956769 \alpha_{\mathrm{s}}+1.482460 \alpha_{\mathrm{s}}^{2}-27.1850 \alpha_{\mathrm{s}}^{3}-53.7325 \alpha_{\mathrm{s}}^{4}+\ldots, \\
& K_{\mathrm{OS}}^{\mathrm{MM}}\left(n_{f}=9\right)=1+3.567723 \alpha_{\mathrm{s}}-1.283997 \alpha_{\mathrm{s}}^{2}-31.8645 \alpha_{\mathrm{s}}^{3}-36.2907 \alpha_{\mathrm{s}}^{4}+\ldots
\end{aligned}
$$



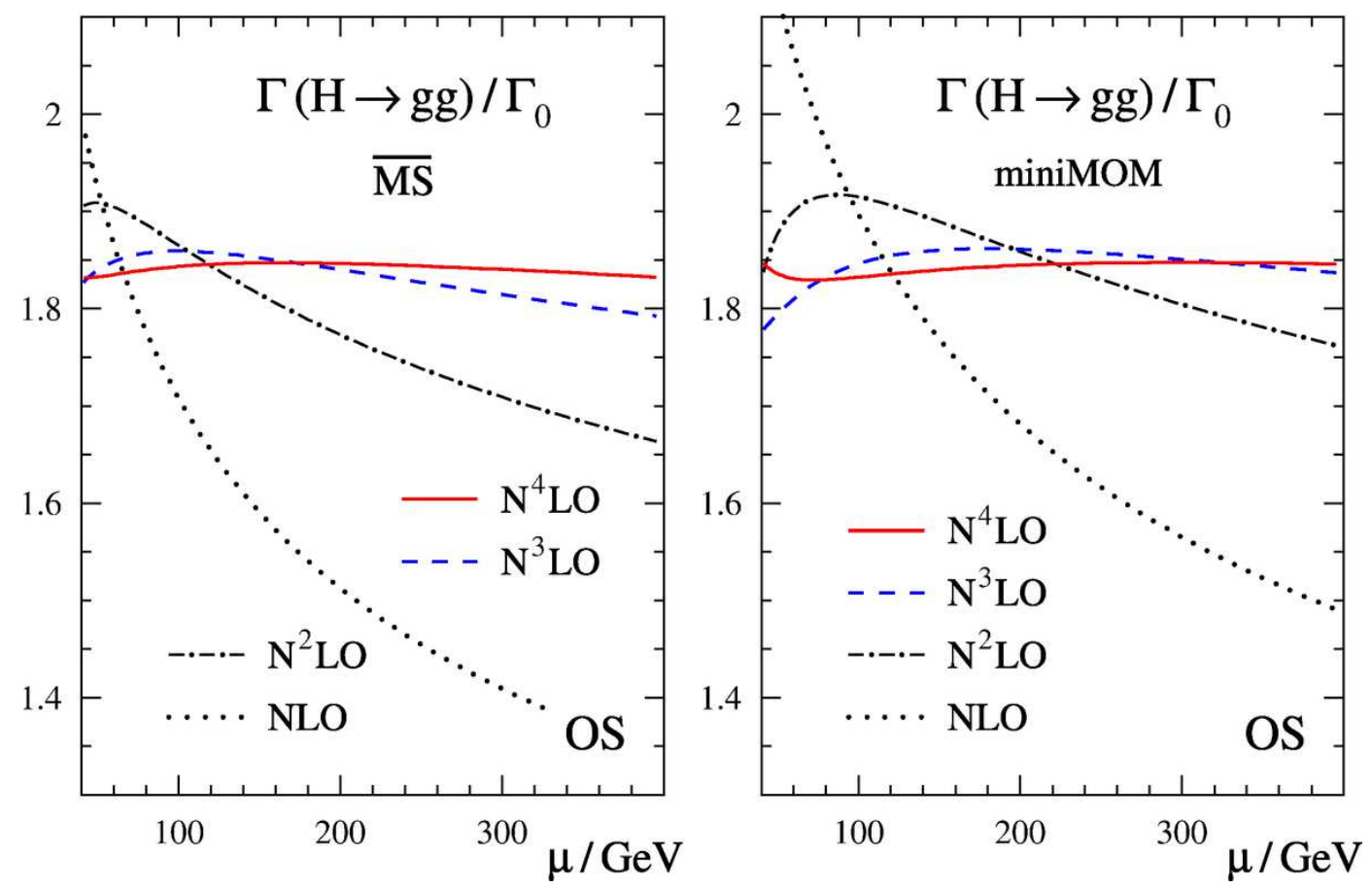

Figure 4. The renormalization scale dependence of the decay width $\Gamma_{H \rightarrow g g}$, normalized as the right part of figure 3, for an on-shell top mass of $173 \mathrm{GeV}$ in $\overline{\mathrm{MS}}$ and the miniMOM scheme.

in terms of $\alpha_{\mathrm{s}}=\alpha_{\mathrm{s}, \mathrm{MM}}$ (here) at $\mu=M_{\mathrm{H}}$ for $M_{t}=173 \mathrm{GeV}$. The miniMOM version of the $n_{f}=5$ OS-scheme expansion coefficients in eq. (3.12) is given by

$$
\begin{aligned}
\gamma_{1, \mathrm{OS}}^{\mathrm{MM}}= & 4.345814-1.220188 L_{H}, \\
\gamma_{2, \mathrm{OS}}^{\mathrm{MM}}= & 4.755361-0.578375 L_{t H}-8.443784 L_{H}+1.116644 L_{H}^{2}, \\
\gamma_{3, \mathrm{OS}}^{\mathrm{MM}}= & -20.15349-2.37892 L_{t H}+0.352863 L_{t H}^{2} \\
& -\left(15.98471-1.41145 L_{t H}\right) L_{H}+10.75116 L_{H}^{2}-0.908344 L_{H}^{3}, \\
\gamma_{4, \mathrm{OS}}^{\mathrm{MM}}= & -72.28293-0.47286 L_{t H}+1.71919 L_{t H}^{2}-0.215280 L_{t H}^{3} \\
& +\left(52.95134+7.82332 L_{t H}+1.07640 L_{t H}^{2}\right) L_{H} \\
& +\left(27.68353-2.15280 L_{t H}\right) L_{H}^{2}-11.29660 L_{H}^{3}+0.692719 L_{H}^{4}
\end{aligned}
$$

The value of the strong coupling in this miniMOM scheme is larger than that in $\overline{\mathrm{MS}}$ with $\alpha_{\mathrm{s}, \mathrm{MM}}\left(M_{\mathrm{Z}}^{2}\right)=1.0960 \alpha_{\mathrm{s}}\left(M_{\mathrm{Z}}^{2}\right)$ for $\alpha_{\mathrm{s}}\left(M_{\mathrm{Z}}^{2}\right)=0.118$ [44], or more generally for $n_{f}=5$ :

$$
\alpha_{\mathrm{s}, \mathrm{MM}}=\alpha_{\mathrm{s}}+0.67862 \alpha_{\mathrm{s}}^{2}+0.91231 \alpha_{\mathrm{s}}^{3}+1.5961 \alpha_{\mathrm{s}}^{4}+3.1629 \alpha_{\mathrm{s}}^{5}+\mathcal{O}\left(\alpha_{\mathrm{s}}^{6}\right) .
$$

This is compensated by lower-order coefficients in eqs. (3.17) and (3.18) that are smaller than their $\overline{\mathrm{MS}}$ counterparts. The $\mathrm{N}^{3} \mathrm{LO}$ and $\mathrm{N}^{4} \mathrm{LO}$ terms for $n_{f}=5$ are not smaller, though.

The resulting perturbative expansion of $\Gamma_{H \rightarrow g g}$ in the Landau-gauge miniMOM scheme is shown in figure 4. The general pattern in miniMOM is somewhat different from that in $\overline{\mathrm{MS}}$ - qualitatively the curves appear shifted to the right. Yet the overall scale range for 
the interval in $\mu$ displayed in the figure is very similar to (if slightly wider than) that in the $\overline{\mathrm{MS}}$ scheme and covered by eq. (3.16). Given this small uncertainty, further investigations of 'optimized scale settings', as performed at $\mathrm{N}^{3} \mathrm{LO}$ in ref. [45] are not warranted.

\section{Higgs decay to bottom quarks}

We denote the perturbative expansion of the function $\widetilde{R}\left(q^{2}\right)$ in eq. (2.12) by

$$
\frac{1}{N_{R}} \widetilde{R}\left(q^{2}\right)=1+\sum_{n=1} \tilde{r}_{n} a_{\mathrm{s}}^{n}\left(q^{2}\right)
$$

in terms of the reduced coupling defined in eq. (2.4). The coefficients up to order $a_{\mathrm{s}}^{4}$ read, for QCD and its generalization to any simple compact gauge group,

$$
\begin{aligned}
\tilde{r}_{1}= & 17 C_{F}, \\
\tilde{r}_{2}= & C_{F}^{2}\left[\frac{691}{4}-36 \zeta_{2}-36 \zeta_{3}\right]+C_{A} C_{F}\left[\frac{893}{4}-22 \zeta_{2}-62 \zeta_{3}\right] \\
& -C_{F} n_{f}\left[\frac{65}{2}-4 \zeta_{2}-8 \zeta_{3}\right], \\
\tilde{r}_{3}= & C_{F}^{3}\left[\frac{23443}{12}-648 \zeta_{2}-956 \zeta_{3}+360 \zeta_{5}\right]+C_{A} C_{F}^{2}\left[\frac{13153}{3}-1532 \zeta_{2}-2178 \zeta_{3}+580 \zeta_{5}\right] \\
& +C_{A}^{2} C_{F}\left[\frac{3894493}{972}-\frac{6860}{9} \zeta_{2}-\frac{4658}{3} \zeta_{3}+\frac{100}{3} \zeta_{5}\right] \\
& -C_{A} C_{F} n_{f}\left[\frac{267800}{243}-\frac{2284}{9} \zeta_{2}-\frac{704}{3} \zeta_{3}+\frac{48}{5} \zeta_{2}^{2}-\frac{80}{3} \zeta_{5}\right] \\
& -C_{F}^{2} n_{f}\left[\frac{2816}{3}-260 \zeta_{2}-520 \zeta_{3}-\frac{48}{5} \zeta_{2}^{2}+160 \zeta_{5}\right]+C_{F} n_{f}^{2}\left[\frac{15511}{243}-\frac{176}{9} \zeta_{2}-16 \zeta_{3}\right] \\
& \left.+21955 \zeta_{5}+\frac{880}{7} \zeta_{2}^{3}+856 \zeta_{3}^{2}+\frac{41517}{4} \zeta_{7}\right] \\
\tilde{r}_{4}= & C_{F}^{4}\left[\frac{915881}{48}-8388 \zeta_{2}-15218 \zeta_{3}+288 \zeta_{2}^{2}+1296 \zeta_{2} \zeta_{3}+7770 \zeta_{5}+768 \zeta_{3}^{2}-1890 \zeta_{7}\right] \\
& +C_{A} C_{F}^{3}\left[\frac{11553691}{144}-\frac{70445}{2} \zeta_{2}-\frac{331541}{6} \zeta_{3}+\frac{7602}{5} \zeta_{2}^{2}+6192 \zeta_{2} \zeta_{3}\right. \\
& \left.+31975 \zeta_{5}+3960 \zeta_{3}^{2}-\frac{32949}{2} \zeta_{7}\right] \\
& C_{A}^{2} C_{F}^{2}\left[\frac{830983045}{7776}-\frac{953327}{18} \zeta_{2}-\frac{450971}{6} \zeta_{3}+\frac{7758}{5} \zeta_{2}^{2}+9724 \zeta_{2} \zeta_{3}\right. \\
& +\frac{672739}{27} \zeta_{2}-\frac{238519}{6} \zeta_{3}+\frac{1739}{3} \zeta_{2}^{2}+\frac{15004}{3} \zeta_{2} \zeta_{3} \\
& +4328 \\
&
\end{aligned}
$$




$$
\begin{aligned}
& \left.-\frac{93875}{9} \zeta_{5}-\frac{880}{7} \zeta_{2}^{3}+5976 \zeta_{3}^{2}-\frac{10899}{4} \zeta_{7}\right] \\
& -\frac{d_{F}^{a b c d} d_{A}^{a b c d}}{N_{R}}\left[144-1300 \zeta_{3}-72 \zeta_{2}^{2}+2440 \zeta_{5}-4896 \zeta_{3}^{2}+1680 \zeta_{7}\right] \\
& +C_{F}^{3} n_{f}\left[-\frac{151297}{9}+6889 \zeta_{2}+\frac{46399}{3} \zeta_{3}-\frac{66}{5} \zeta_{2}^{2}-1584 \zeta_{2} \zeta_{3}\right. \\
& \left.-9380 \zeta_{5}-\frac{480}{7} \zeta_{2}^{3}-1368 \zeta_{3}^{2}+3360 \zeta_{7}\right] \\
& -C_{A} C_{F}^{2} n_{f}\left[\frac{83380613}{1944}-\frac{162944}{9} \zeta_{2}-\frac{79736}{3} \zeta_{3}+\frac{3444}{5} \zeta_{2}^{2}+3128 \zeta_{2} \zeta_{3}\right. \\
& \left.+\frac{23314}{3} \zeta_{5}-\frac{80}{7} \zeta_{2}^{3}+652 \zeta_{3}^{2}+1680 \zeta_{7}\right] \\
& -C_{A}^{2} C_{F} n_{f}\left[\frac{72695765}{1944}-11949 \zeta_{2}-\frac{158515}{18} \zeta_{3}+\frac{2123}{5} \zeta_{2}^{2}+1936 \zeta_{2} \zeta_{3}\right. \\
& \left.-\frac{65812}{9} \zeta_{5}-\frac{400}{7} \zeta_{2}^{3}+700 \zeta_{3}^{2}-280 \zeta_{7}\right] \\
& +\frac{d_{F}^{a b c d} d_{F}^{a b c d}}{N_{R}} n_{f}\left[348-2008 \zeta_{3}-144 \zeta_{2}^{2}-1152 \zeta_{3}^{2}+2560 \zeta_{5}\right] \\
& +C_{F}^{2} n_{f}^{2}\left[\frac{7009861}{1944}-\frac{13210}{9} \zeta_{2}-\frac{8146}{3} \zeta_{3}+\frac{204}{5} \zeta_{2}^{2}+352 \zeta_{2} \zeta_{3}+192 \zeta_{3}^{2}+\frac{2848}{3} \zeta_{5}\right] \\
& +C_{A} C_{F} n_{f}^{2}\left[\frac{18248293}{3888}-\frac{16031}{9} \zeta_{2}-\frac{4972}{9} \zeta_{3}+\frac{324}{5} \zeta_{2}^{2}+304 \zeta_{2} \zeta_{3}-32 \zeta_{3}^{2}-\frac{10484}{9} \zeta_{5}\right] \\
& -C_{F} n_{f}^{3}\left[\frac{520771}{2916}-\frac{2200}{27} \zeta_{2}-\frac{260}{9} \zeta_{3}+\frac{8}{3} \zeta_{2}^{2}+\frac{64}{3} \zeta_{2} \zeta_{3}-\frac{160}{3} \zeta_{5}\right] .
\end{aligned}
$$

Eqs. (4.2)-(4.4) agree with the literature, see refs. [56, 57] and references therein. The $\mathrm{N}^{4} \mathrm{LO}$ coefficient $\tilde{r}_{4}$ has been computed in ref. [4] for the case of QCD. Accordingly setting $C_{A}=3, C_{F}=4 / 3, d_{F}^{a b c d} d_{A}^{a b c d} / N_{R}=5 / 2$ and $d_{F}^{a b c d} d_{F}^{a b c d} / N_{R}=5 / 36$ in eq. (4.5), we find complete agreement with their result.

Only the case of $n_{f}=5$ is phenomenologically relevant, yet it is instructive to briefly consider the numerical dependence of $\widetilde{R}$ on the number of light flavours $n_{f}$ over a wide range,

$$
\begin{aligned}
& n_{f}=1: 1+1.8037560 \alpha_{\mathrm{s}}+3.5038193 \alpha_{\mathrm{s}}^{2}+4.470933 \alpha_{\mathrm{s}}^{3}-1.765010 \alpha_{\mathrm{s}}^{4}+\ldots, \\
& n_{f}=2: 1+1.8037560 \alpha_{\mathrm{s}}+3.3661592 \alpha_{\mathrm{s}}^{2}+3.664830 \alpha_{\mathrm{s}}^{3}-3.736264 \alpha_{\mathrm{s}}^{4}+\ldots, \\
& n_{f}=3: 1+1.8037560 \alpha_{\mathrm{s}}+3.2284991 \alpha_{\mathrm{s}}^{2}+2.875431 \alpha_{\mathrm{s}}^{3}-5.511190 \alpha_{\mathrm{s}}^{4}+\ldots, \\
& n_{f}=4: 1+1.8037560 \alpha_{\mathrm{s}}+3.0908390 \alpha_{\mathrm{s}}^{2}+2.102737 \alpha_{\mathrm{s}}^{3}-7.091048 \alpha_{\mathrm{s}}^{4}+\ldots, \\
& n_{f}=5: 1+1.8037560 \alpha_{\mathrm{s}}+2.9531789 \alpha_{\mathrm{s}}^{2}+1.346747 \alpha_{\mathrm{s}}^{3}-8.477010 \alpha_{\mathrm{s}}^{4}+\ldots, \\
& n_{f}=6: 1+1.8037560 \alpha_{\mathrm{s}}+2.8155188 \alpha_{\mathrm{s}}^{2}+0.607462 \alpha_{\mathrm{s}}^{3}-9.670604 \alpha_{\mathrm{s}}^{4}+\ldots .
\end{aligned}
$$


The main trend is similar to that of the larger coefficients for $\Gamma_{H \rightarrow g g}$ in eqs. (3.10) and (3.11): the $n_{f}$-dependent coefficients decrease with increasing $n_{f}$. The main difference is that the fourth-order term is already negative at $n_{f}=1$. The $\alpha_{\mathrm{s}}^{3}$-term changes sign close to $n_{f}=7$, leading to the large fourth-order / third-order ratio at $n_{f}=5$ already observed in ref. [4].

The break-up of the coefficients $\tilde{r}$ for QCD into 'genuine' and 'kinematic' contributions can be found in eq. (7) of ref. [4]. The numerical scale dependence of $\widetilde{R}$ has been included in the comprehensive study of Higgs decays to hadrons to order $\alpha_{\mathrm{s}}^{4}$ in ref. [3]. However, the scale dependence of the coefficients $\tilde{r}_{n}$ is available in the literature only to order $\alpha_{\mathrm{s}}^{3}$ [57].

For the convenience of the reader, we therefore conclude our brief account of $\Gamma_{H \rightarrow \bar{b}} b$ by writing down the generalization of the coefficients (4.2)-(4.5) to a general scale $\mu^{2}$ :

$$
\begin{aligned}
\tilde{r}_{1}\left(L_{q}\right)=\tilde{r}_{1} & -2 \gamma_{0} L_{q}, \\
\tilde{r}_{2}\left(L_{q}\right)=\tilde{r}_{2} & -\left(2 \gamma_{1}+2 \tilde{r}_{1} \gamma_{1}+\tilde{r}_{1} \beta_{0}\right) L_{q}+\left(2 \gamma_{0}^{2}+\beta_{0} \gamma_{0}\right) L_{q}^{2}, \\
\tilde{r}_{3}\left(L_{q}\right)=\tilde{r}_{3}- & \left(2 \gamma_{2}+2 \tilde{r}_{2} \gamma_{0}+2 \tilde{r}_{2} \beta_{0}+2 \tilde{r}_{1} \gamma_{1}+\tilde{r}_{1} \beta_{1}\right) L_{q} \\
& +\left(4 \gamma_{0} \gamma_{1}+\beta_{1} \gamma_{0}+2 \beta_{0} \gamma_{1}+2 \tilde{r}_{1} \gamma_{0}^{2}+3 \tilde{r}_{1} \beta_{0} \gamma_{0}+\tilde{r}_{1} \beta_{0}^{2}\right) L_{q}^{2} \\
& -1 / 3\left(4 \gamma_{0}^{3}+6 \beta_{0} \gamma_{0}^{2}+2 \beta_{0}^{2} \gamma_{0}\right) L_{q}^{3}, \\
\tilde{r}_{4}\left(L_{q}\right)=\tilde{r}_{4} & -\left(2 \gamma_{3}+2 \tilde{r}_{3} \gamma_{0}+3 \tilde{r}_{3} \beta_{0}+2 \tilde{r}_{2} \gamma_{1}+2 \tilde{r}_{2} \beta_{1}+2 \tilde{r}_{1} \gamma_{2}+\tilde{r}_{1} \beta_{2}\right) L_{q} \\
+ & \left(2 \gamma_{1}^{2}+4 \gamma_{0} \gamma_{2}+\beta_{2} \gamma_{0}+2 \beta_{1} \gamma_{1}+3 \beta_{0} \gamma_{2}+2 \tilde{r}_{2} \gamma_{0}^{2}+5 \tilde{r}_{2} \beta_{0} \gamma_{0}\right. \\
+ & \left.3 \tilde{r}_{2} \beta_{0}^{2}+4 \tilde{r}_{1} \gamma_{0} \gamma_{1}+3 \tilde{r}_{1} \beta_{1} \gamma_{0}+4 \tilde{r}_{1} \beta_{0} \gamma_{1}+5 / 2 \tilde{r}_{1} \beta_{0} \beta_{1}\right) L_{q}^{2} \\
& -1 / 3\left(12 \gamma_{0}^{2} \gamma_{1}+6 \beta_{1} \gamma_{0}^{2}+18 \beta_{0} \gamma_{0} \gamma_{1}+5 \beta_{0} \beta_{1} \gamma_{0}+6 \beta_{0}^{2} \gamma_{1}\right. \\
& \left.+4 \tilde{r}_{1} \gamma_{0}^{3}+12 \tilde{r}_{1} \beta_{0} \gamma_{0}^{2}+11 \tilde{r}_{1} \beta_{0}^{2} \gamma_{0}+3 \tilde{r}_{1} \beta_{0}^{3}\right) L_{q}^{3} \\
+ & 1 / 6\left(4 \gamma_{0}^{4}+12 \beta_{0} \gamma_{0}^{3}+11 \beta_{0}^{2} \gamma_{0}^{2}+3 \beta_{0}^{3} \gamma_{0}\right) L_{q}^{4},
\end{aligned}
$$

in terms of $L_{q}=\ln \left(q^{2} / \mu^{2}\right), \tilde{r}_{n}$ in eqs. (4.2)-(4.3), the coefficients $\beta_{n}$ of the beta function, and the coefficients $\gamma_{n}$ of the mass anomalous dimension in the $\overline{\mathrm{MS}}$ scheme up to $\mathrm{N}^{3} \mathrm{LO}$, see refs. $[58,59]$ and references therein. The coefficients to $\tilde{r}_{3}\left(L_{q}\right)$ agree with eq. (17) of ref. [57].

\section{The electromagnetic R-ratio}

The non-singlet and singlet contributions to the electromagnetic R-ratio, $r\left(q^{2}\right)$ and $r_{\mathrm{S}}\left(q^{2}\right)$ in eq. (2.15), can be expanded in the same manner as $N_{R}^{-1} \widetilde{R}\left(q^{2}\right)$ in eq. (4.1). At $\mu^{2}=q^{2}$ the coefficients for the dominant non-singlet part read

$$
\begin{aligned}
& r_{1}=3 C_{F}, \\
& r_{2}=-\frac{3}{2} C_{F}^{2}+C_{A} C_{F}\left[\frac{123}{2}-44 \zeta_{3}\right]-C_{F} n_{f}\left[11-8 \zeta_{3}\right],
\end{aligned}
$$




$$
\begin{aligned}
& r_{3}=-\frac{69}{2} C_{F}^{3}-C_{A} C_{F}^{2}\left[127+572 \zeta_{3}-880 \zeta_{5}\right] \\
& +C_{A}^{2} C_{F}\left[\frac{90445}{54}-\frac{242}{3} \zeta_{2}-\frac{10948}{9} \zeta_{3}-\frac{440}{3} \zeta_{5}\right]-C_{F}^{2} n_{f}\left[\frac{29}{2}-152 \zeta_{3}+160 \zeta_{5}\right] \\
& -C_{A} C_{F} n_{f}\left[\frac{15520}{27}-\frac{88}{3} \zeta_{2}-\frac{3584}{9} \zeta_{3}-\frac{80}{3} \zeta_{5}\right]+C_{F} n_{f}^{2}\left[\frac{1208}{27}-\frac{8}{3} \zeta_{2}-\frac{304}{9} \zeta_{3}\right] \text {, } \\
& r_{4}=C_{F}^{4}\left[\frac{4157}{8}+96 \zeta_{3}\right]-C_{A} C_{F}^{3}\left[2024+278 \zeta_{3}-18040 \zeta_{5}+18480 \zeta_{7}\right] \\
& -C_{A}^{2} C_{F}^{2}\left[\frac{592141}{72}-121 \zeta_{2}+\frac{87850}{3} \zeta_{3}-\frac{104080}{3} \zeta_{5}-9240 \zeta_{7}\right] \\
& +C_{A}^{3} C_{F}\left[\frac{52207039}{972}-\frac{16753}{3} \zeta_{2}-\frac{912446}{27} \zeta_{3}+\frac{10648}{3} \zeta_{2} \zeta_{3}-\frac{155990}{9} \zeta_{5}\right. \\
& \left.+4840 \zeta_{3}^{2}-1540 \zeta_{7}\right] \\
& +\frac{d_{F}^{a b c d} d_{A}^{a b c d}}{N_{R}}\left[48-64 \zeta_{3}-320 \zeta_{5}\right]-n_{f} \frac{d_{F}^{a b c d} d_{F}^{a b c d}}{N_{R}}\left[208+256 \zeta_{3}-640 \zeta_{5}\right] \\
& +C_{F}^{3} n_{f}\left[\frac{1001}{3}+396 \zeta_{3}-4000 \zeta_{5}+3360 \zeta_{7}\right] \\
& +C_{A} C_{F}^{2} n_{f}\left[\frac{32357}{108}+66 \zeta_{2}+\frac{42644}{3} \zeta_{3}-\frac{41240}{3} \zeta_{5}-1056 \zeta_{3}^{2}-1680 \zeta_{7}\right] \\
& -C_{A}^{2} C_{F} n_{f}\left[\frac{4379861}{162}-2988 \zeta_{2}-\frac{137744}{9} \zeta_{3}+1936 \zeta_{2} \zeta_{3}-\frac{75220}{9} \zeta_{5}+704 \zeta_{3}^{2}-280 \zeta_{7}\right] \\
& +C_{F}^{2} n_{f}^{2}\left[\frac{5713}{27}-16 \zeta_{2}-\frac{4648}{3} \zeta_{3}+\frac{4000}{3} \zeta_{5}+192 \zeta_{3}^{2}\right] \\
& +C_{A} C_{F} n_{f}^{2}\left[\frac{340843}{81}-520 \zeta_{2}-\frac{20906}{9} \zeta_{3}+352 \zeta_{2} \zeta_{3}-\frac{10880}{9} \zeta_{5}-32 \zeta_{3}^{2}\right] \\
& -C_{F} n_{f}^{3}\left[\frac{49048}{243}-\frac{88}{3} \zeta_{2}-\frac{3248}{27} \zeta_{3}+\frac{64}{3} \zeta_{2} \zeta_{3}-\frac{160}{3} \zeta_{5}\right] \text {. }
\end{aligned}
$$

Additional singlet contributions enter from the third order in $\alpha_{\mathrm{s}}$, viz

$$
\begin{aligned}
r_{3, S}= & \frac{d_{F}^{a b c} d_{F}^{a b c}}{N_{R}}\left[\frac{176}{3}-128 \zeta_{3}\right] \\
r_{4, S}= & \frac{d_{F}^{a b c} d_{F}^{a b c}}{N_{R}}\left(C_{A}\left[\frac{31144}{9}-5408 \zeta_{3}+2880 \zeta_{5}-1408 \zeta_{3}^{2}\right]\right. \\
& \left.-C_{F}\left[832+1024 \zeta_{3}-2560 \zeta_{5}\right]-n_{f}\left[\frac{4768}{9}-832 \zeta_{3}+640 \zeta_{5}-256 \zeta_{3}^{2}\right]\right)
\end{aligned}
$$


with $d_{F}^{a b c} d_{F}^{a b c} / N_{R}=5 / 18$ in QCD; for the 'time-dependent' normalization of this colour factor see the discussion below eq. (30) of ref. [62]. The above results are in complete agreement with previous calculations, see refs. $[19-22,60,61]$ and references therein. The fourth-order coefficients (5.4) and (5.6) had not been verified by a second calculation before.

The numerical expansion of the non-singlet contribution $r\left(q^{2}\right)$ in QCD is given by

$$
\begin{aligned}
& n_{f}=1: 1+0.3183099 \alpha_{\mathrm{s}}+0.1895124 \alpha_{\mathrm{s}}^{2}-0.252925 \alpha_{\mathrm{s}}^{3}-1.422960 \alpha_{\mathrm{s}}^{4}+\ldots, \\
& n_{f}=2: 1+0.3183099 \alpha_{\mathrm{s}}+0.1778305 \alpha_{\mathrm{s}}^{2}-0.213173 \alpha_{\mathrm{s}}^{3}-1.253232 \alpha_{\mathrm{s}}^{4}+\ldots, \\
& n_{f}=3: 1+0.3183099 \alpha_{\mathrm{s}}+0.1661486 \alpha_{\mathrm{s}}^{2}-0.331673 \alpha_{\mathrm{s}}^{3}-1.097226 \alpha_{\mathrm{s}}^{4}+\ldots, \\
& n_{f}=4: 1+0.3183099 \alpha_{\mathrm{s}}+0.1544668 \alpha_{\mathrm{s}}^{2}-0.371548 \alpha_{\mathrm{s}}^{3}-0.953617 \alpha_{\mathrm{s}}^{4}+\ldots, \\
& n_{f}=5: 1+0.3183099 \alpha_{\mathrm{s}}+0.1427849 \alpha_{\mathrm{s}}^{2}-0.411757 \alpha_{\mathrm{s}}^{3}-0.821078 \alpha_{\mathrm{s}}^{4}+\ldots, \\
& n_{f}=6: 1+0.3183099 \alpha_{\mathrm{s}}+0.1311030 \alpha_{\mathrm{s}}^{2}-0.452301 \alpha_{\mathrm{s}}^{3}-0.698289 \alpha_{\mathrm{s}}^{4}+\ldots .
\end{aligned}
$$

The physically relevant numbers of effectively massless flavours are $n_{f}=3, \ldots, 6$. The overall effect of the quartic group invariants on the fourth-order coefficient is between $3 \%$ and $5 \%$ for these values of $n_{f}$.

The $\alpha_{\mathrm{s}}$-corrections in eq. (5.7) are much smaller than their counterparts for $H \rightarrow g g$ in eq. (3.7) and $H \rightarrow \bar{b} b$ in eq. (4.6); as in the latter case the $n_{f}$-dependent coefficients decrease with increasing $n_{f}$. The fourth-order correction is largest for low values of $n_{f}$ : $r_{4}$ amounts to 5.6 times $r_{3}$ at $n_{f}=1$. For three flavours the $\alpha_{\mathrm{s}}^{4}$ correction contributes as much as the $\alpha_{\mathrm{s}}^{3}$ terms at $\alpha_{\mathrm{s}} \simeq 0.3$. This situation is at least exacerbated by the kinematic $\pi^{2}$ contributions, as can be seen from the example decompositions

$$
\begin{aligned}
& n_{f}=1: \ldots+(0.4551676-\underline{0.7080921}) \alpha_{\mathrm{s}}^{3}+(1.0596193-\underline{2.4825797}) \alpha_{\mathrm{s}}^{4}+\ldots \\
& n_{f}=3: \ldots+(0.2054750-\underline{0.5371479}) \alpha_{\mathrm{s}}^{3}+(0.5038103-\underline{1.6010363}) \alpha_{\mathrm{s}}^{4}+\ldots
\end{aligned}
$$

where, as above, those contributions have been underlined. For the full $n_{f}$-dependence of this decomposition see eq. (7) of ref. [19].

The generalization of the coefficients in eqs. (5.1)-(5.4) to $\mu^{2} \neq q^{2}$ can be obtained from eqs. (4.7) by dropping the terms with $\gamma_{n}$ which arise from the Yukawa-coupling prefactor $m_{b}^{2} \equiv m_{b}^{2}\left(\mu^{2}\right)$ in eq. (2.12). The resulting numerical dependence of $r\left(q^{2}\right)$ is very small at the particularly important point $q^{2}=M_{\mathrm{Z}}^{2}$, see figures 2 and 3 of ref. [21].

The transformation of eqs. (5.7) to the miniMOM scheme yields, with $\alpha_{\mathrm{s}}=\alpha_{\mathrm{s}, \mathrm{MM}}$ (here),

$$
\begin{aligned}
& n_{f}=1: 1+0.3183099 \alpha_{\mathrm{s}}-0.1390779 \alpha_{\mathrm{s}}^{2}-0.780651 \alpha_{\mathrm{s}}^{3}-0.511193 \alpha_{\mathrm{s}}^{4}+\ldots, \\
& n_{f}=2: 1+0.3183099 \alpha_{\mathrm{s}}-0.1226150 \alpha_{\mathrm{s}}^{2}-0.736947 \alpha_{\mathrm{s}}^{3}-0.342317 \alpha_{\mathrm{s}}^{4}+\ldots, \\
& n_{f}=3: 1+0.3183099 \alpha_{\mathrm{s}}-0.1061521 \alpha_{\mathrm{s}}^{2}-0.692733 \alpha_{\mathrm{s}}^{3}-0.190425 \alpha_{\mathrm{s}}^{4}+\ldots, \\
& n_{f}=4: 1+0.3183099 \alpha_{\mathrm{s}}-0.0896891 \alpha_{\mathrm{s}}^{2}-0.648007 \alpha_{\mathrm{s}}^{3}-0.054783 \alpha_{\mathrm{s}}^{4}+\ldots, \\
& n_{f}=5: 1+0.3183099 \alpha_{\mathrm{s}}-0.0732262 \alpha_{\mathrm{s}}^{2}-0.602769 \alpha_{\mathrm{s}}^{3}+0.065345 \alpha_{\mathrm{s}}^{4}+\ldots, \\
& n_{f}=6: 1+0.3183099 \alpha_{\mathrm{s}}-0.0567633 \alpha_{\mathrm{s}}^{2}-0.557020 \alpha_{\mathrm{s}}^{3}+0.170696 \alpha_{\mathrm{s}}^{4}+\ldots,
\end{aligned}
$$



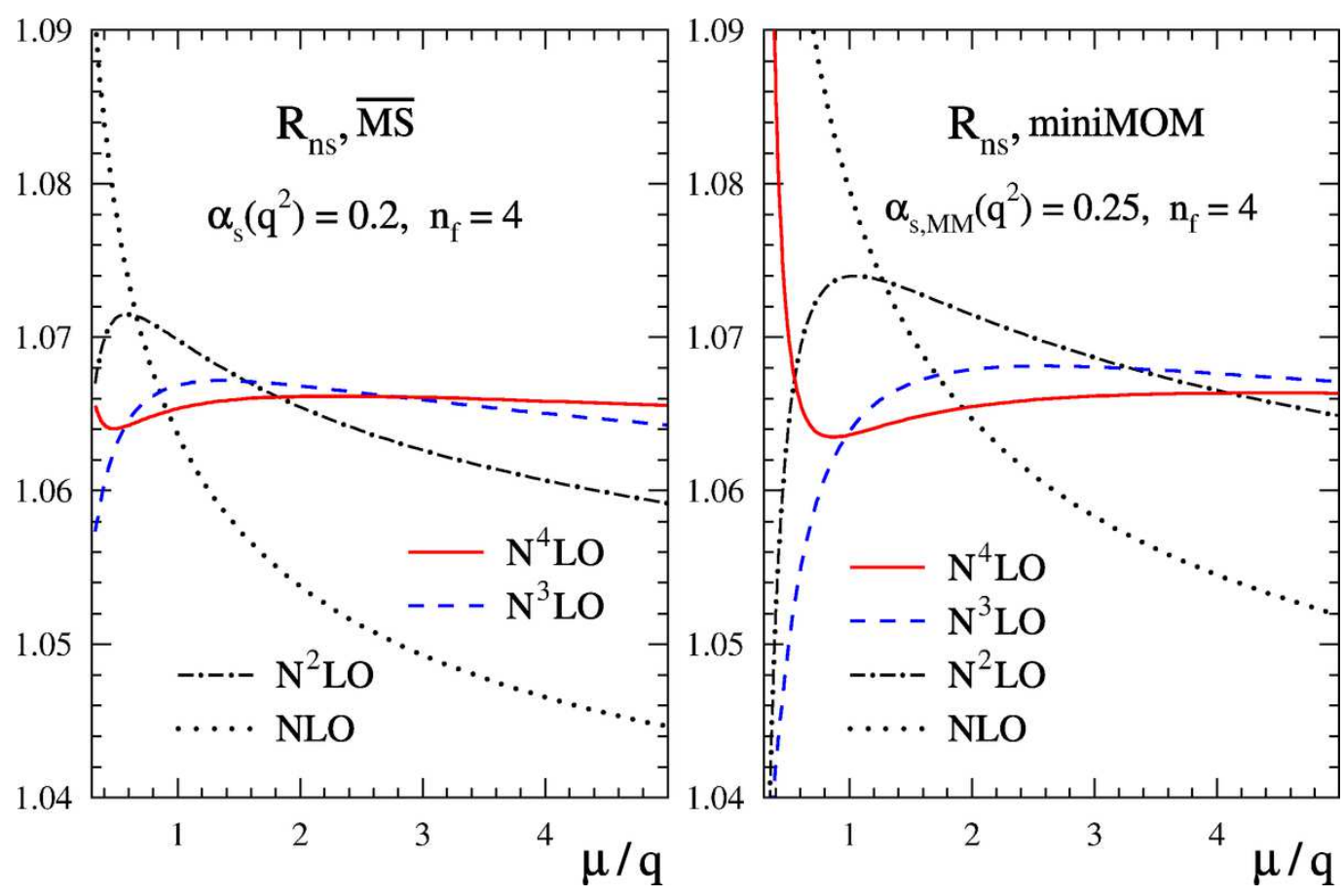

Figure 5. The renormalization scale dependence of the non-singlet $R$-ratio for $n_{f}=4$ at a reference scale, specified by $\alpha_{\mathrm{s}}\left(q^{2}\right)=0.2$ in $\overline{\mathrm{MS}}$, below the $\Upsilon$ threshold in the $\overline{\mathrm{MS}}$ and miniMOM schemes.

in agreement with the corresponding parts of eq. (3.5)-(3.10) in ref. [63] (see also ref. [64]), where the expansion has been written down in terms of $a_{\mathrm{s}}=\alpha_{\mathrm{s}} /(4 \pi)$. The qualitative pattern in eq. (5.9) is rather different from that in eq. (5.7): here the ratios of the thirdorder and second-order coefficients are large. If the fourth-order results were not known, one might by tempted to expect a further rapid growth of the coefficients at this order. Yet, the actual numbers are much smaller than their third-order counterparts for the physical values of $n_{f}$.

The generalization of eq. (5.9) to $\mu^{2} \neq q^{2}$ is again given by eqs. (4.7) with $\gamma_{n}=0$, but with the $\overline{\mathrm{MS}}$ beta function replaced by its miniMOM counterpart [42, 43]. The $\mu$ dependence of the $R$-ratio up to order $\alpha_{\mathrm{s}}^{4}$ is shown for both schemes in figures 5 and 6 at two low- $q^{2}$ reference points. The first is above the $\bar{c} c$ resonances but below the $\Upsilon$ threshold, where an analysis with $n_{f}=4$ is appropriate [21]. The second is below the $J / \psi$ resonance with $n_{f}=3$.

The respective scales are specified via (order-independent) $\overline{\mathrm{MS}}$ values of $\alpha_{\mathrm{s}}\left(q^{2}\right)$, the corresponding miniMOM values of $\alpha_{\mathrm{S}}$ are rounded results of the $\mathrm{N}^{4} \mathrm{LO}$ conversion of ref. [44]. The $\overline{\mathrm{MS}}$ scale variation in figure 6 amounts to $R_{\mathrm{ns}}-1=(6.51 \pm 0.11) \cdot 10^{-2}$ at $\mathrm{N}^{4} \mathrm{LO}$. The corresponding miniMOM band is consistent with this result, and only slightly wider if the small- $\mu$ spike is not taken into account. For a very low $q^{2}$ with $\alpha_{\mathrm{s}}\left(q^{2}\right)=0.3$, the results become unstable below about $\mu=q$ in $\overline{\mathrm{MS}}$ and $\mu=2 q$ in miniMOM. Disregarding these regions, the $\mathrm{N}^{4} \mathrm{LO}$ results are fairly stable with a $3 \%$ uncertainty and $R_{\mathrm{ns}}-1=(9.5 \pm 0.3) \cdot 10^{-2}$ in the $\overline{\mathrm{MS}}$ scheme. 

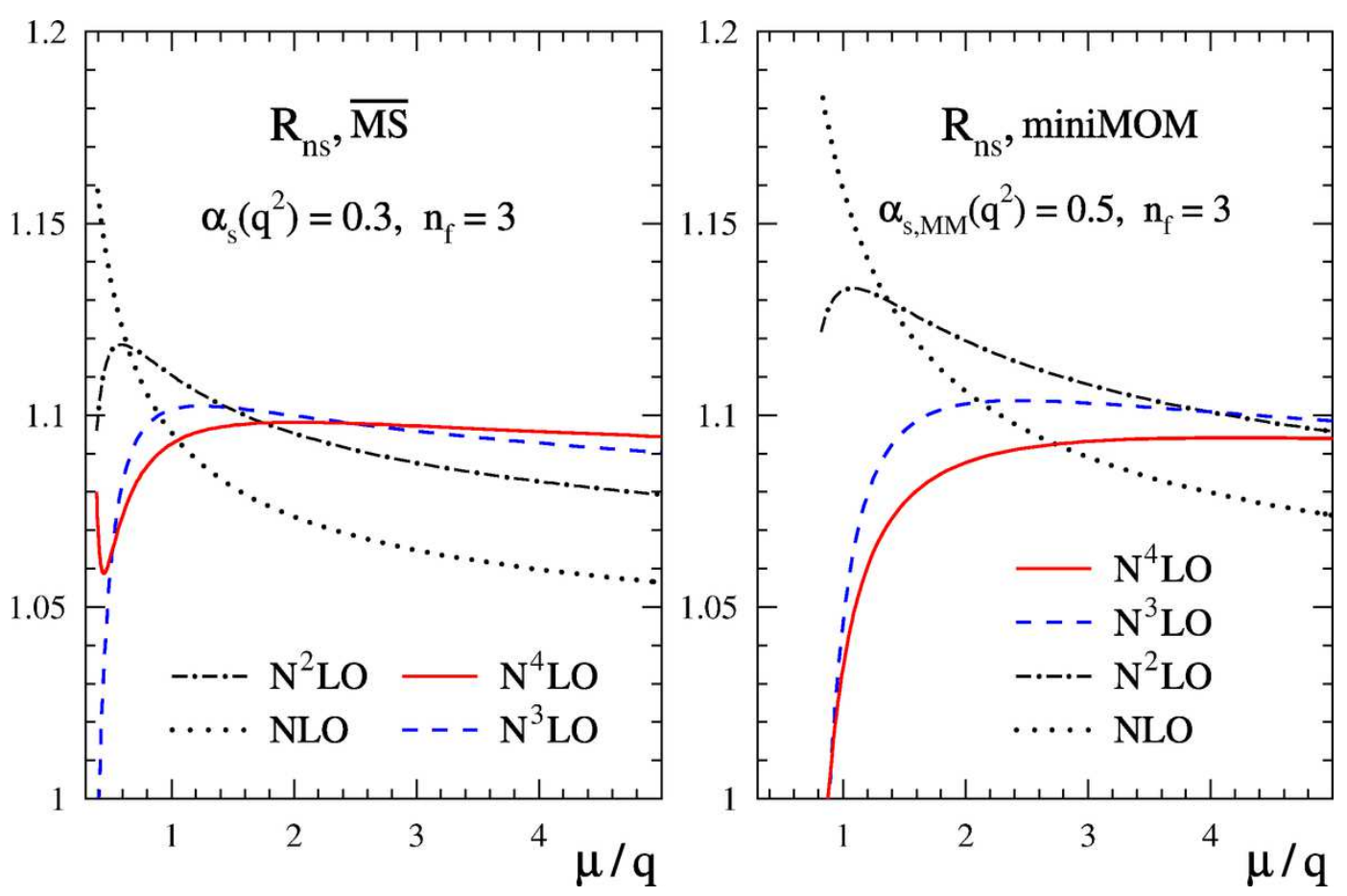

Figure 6. As figure 5 , but for a scale below the $J / \psi$ threshold with $\alpha_{\mathrm{s}}=0.3$ in $\overline{\mathrm{MS}}$ and $n_{f}=3$. The curves have been cut off at low scales where the respective values of $\alpha_{\mathrm{s}}$ at $\mathrm{N}^{3} \mathrm{LO}$ exceed 0.7.

\section{Summary}

We have completed the $\mathrm{N}^{4} \mathrm{LO}$ corrections, i.e., the contributions of order $\alpha_{\mathrm{s}}^{6}$, for the decay of the Higgs boson to hadrons via $H \rightarrow g g$ at the leading order in the heavy-top limit. This correction is slightly smaller than the $1 / m_{\text {top }}$ effects at NNLO [7] but, in all likelihood, larger than the presently unknown $1 / m_{\text {top }}$ correction at $\mathrm{N}^{3} \mathrm{LO}$. Hence our result provides an improvement of the overall accuracy of $\Gamma_{H \rightarrow g g}$. The remaining uncertainty due to the truncation of the perturbation series can be estimated, rather conservatively, as $\pm 0.6 \%$. This is much smaller than the uncertainty of $2.5 \%$ induced by a $1 \%$ uncertainty of $\alpha_{\mathrm{S}}\left(M_{\mathrm{Z}}^{2}\right)$. An experimental uncertainty of $1 \%$ is, of course, not feasible at the LHC. However, a future linear $e^{+} e^{-}$collider may be able to address the coupling of the Higgs boson to gluons at this level, see section 2.3 of ref. [65].

Furthermore we have calculated, also for a general gauge group, the fourth-order corrections to $H \rightarrow \bar{b} b$ in the massless limit and to the electromagnetic $R$-ratio for $e^{+} e^{-} \rightarrow$ hadrons. These corrections have been computed by one group before in refs. [4] (where the result is presented only for the gauge group $\mathrm{SU}(3)$ ) and [19-22], respectively; we find complete agreement with those results.

Our calculations have been performed using the FORCER program [28] and, at five loops, a Form implementation of the $R^{*}$-methods introduced very recently in ref. [32]. These methods differ substantially from the global $R^{*}$-method used in refs. [4, 19-22, 38]. Up to four loops we were easily able to keep all powers of the gauge parameter. This was prohibitively expensive at the five-loop level where we worked in the Feynman gauge. 
These results have been checked in two ways. The first is verifying the correctness of the higher poles in $\varepsilon$, which have to cancel against the effect of lower-order diagrams in the renormalization procedure. The second check is the computation of all five-loop diagrams in at least two different ways.

We have illustrated the size and renormalization-scale dependence of $\Gamma_{H \rightarrow g g}$ and the $R$-ratio in both the standard $\overline{\mathrm{MS}}$ scheme and the miniMOM scheme. The $\mathrm{N}^{4} \mathrm{LO}$ results and their stability in these two schemes are comparable for $\Gamma_{H \rightarrow g g}$ and $R$-ratio at high scales $q^{2}$; the miniMOM results for the $R$-ratio at renormalization scale $\mu^{2} \simeq q^{2}$ become unstable at higher $q^{2}$ than their $\overline{\mathrm{MS}}$ counterparts. Overall the miniMOM scheme does not appear to be preferable over $\overline{\mathrm{MS}}$ in cases, such as the ones considered here, where the perturbation series is known to a high order.

Form files with our results can be obtained from the preprint server http://arXiv.org by downloading the source of this article. They are also available from the authors upon request.

\section{Acknowledgments}

This work has been supported by the European Research Council (ERC) Advanced Grant 320651, HEPGAME and the U.K. Science 8 Technology Facilities Council (STFC) grant ST/L000431/1. We also are grateful for the opportunity to use most of the ulgqcd computer cluster in Liverpool which was funded by the STFC grant ST/H008837/1. The Feynman-diagram figures were made using Axodraw2 [66].

Open Access. This article is distributed under the terms of the Creative Commons Attribution License (CC-BY 4.0), which permits any use, distribution and reproduction in any medium, provided the original author(s) and source are credited.

\section{References}

[1] ATLAS collaboration, Observation of a new particle in the search for the Standard Model Higgs boson with the ATLAS detector at the LHC, Phys. Lett. B 716 (2012) 1 [arXiv: 1207.7214] [INSPIRE].

[2] CMS collaboration, Observation of a new boson at a mass of $125 \mathrm{GeV}$ with the CMS experiment at the LHC, Phys. Lett. B 716 (2012) 30 [arXiv:1207.7235] [INSPIRE].

[3] J. Davies, M. Steinhauser and D. Wellmann, Completing the hadronic Higgs boson decay at order $\alpha_{\mathrm{s}}^{4}$, Nucl. Phys. B 920 (2017) 20 [arXiv:1703.02988] [INSPIRE].

[4] P.A. Baikov, K.G. Chetyrkin and J.H. Kuhn, Scalar correlator at $O\left(\alpha_{\mathrm{s}}^{4}\right)$, Higgs decay into b-quarks and bounds on the light quark masses, Phys. Rev. Lett. 96 (2006) 012003 [hep-ph/0511063] [INSPIRE].

[5] T. Inami, T. Kubota and Y. Okada, Effective Gauge Theory and the Effect of Heavy Quarks in Higgs Boson Decays, Z. Phys. C 18 (1983) 69 [INSPIRE].

[6] S.A. Larin, T. van Ritbergen and J.A.M. Vermaseren, The Large top quark mass expansion for Higgs boson decays into bottom quarks and into gluons, Phys. Lett. B 362 (1995) 134 [hep-ph/9506465] [INSPIRE]. 
[7] M. Schreck and M. Steinhauser, Higgs Decay to Gluons at NNLO, Phys. Lett. B 655 (2007) 148 [arXiv: 0708.0916] [InSPIRE].

[8] M. Krämer, E. Laenen and M. Spira, Soft gluon radiation in Higgs boson production at the LHC, Nucl. Phys. B 511 (1998) 523 [hep-ph/9611272] [INSPIRE].

[9] K.G. Chetyrkin, B.A. Kniehl and M. Steinhauser, Decoupling relations to $O\left(\alpha_{\mathrm{s}}^{3}\right)$ and their connection to low-energy theorems, Nucl. Phys. B 510 (1998) 61 [hep-ph/9708255] [INSPIRE].

[10] Y. Schröder and M. Steinhauser, Four-loop decoupling relations for the strong coupling, JHEP 01 (2006) 051 [hep-ph/0512058] [INSPIRE].

[11] K.G. Chetyrkin, J.H. Kühn and C. Sturm, QCD decoupling at four loops, Nucl. Phys. B 744 (2006) 121 [hep-ph/0512060] [INSPIRE].

[12] B.A. Kniehl, A.V. Kotikov, A.I. Onishchenko and O.L. Veretin, Strong-coupling constant with flavor thresholds at five loops in the anti-MS scheme, Phys. Rev. Lett. 97 (2006) 042001 [hep-ph/0607202] [INSPIRE].

[13] K. Chetyrkin, P. Baikov and J. Kühn, The $\beta$-function of Quantum Chromodynamics and the effective Higgs-gluon-gluon coupling in five-loop order, PoS (LL2016) 010.

[14] P.A. Baikov and K.G. Chetyrkin, Top Quark Mediated Higgs Boson Decay into Hadrons to Order $\alpha_{\mathrm{s}}^{5}$, Phys. Rev. Lett. 97 (2006) 061803 [hep-ph/0604194] [INSPIRE].

[15] S. Moch and A. Vogt, On third-order timelike splitting functions and top-mediated Higgs decay into hadrons, Phys. Lett. B 659 (2008) 290 [arXiv:0709.3899] [INSPIRE].

[16] J. Davies, M. Steinhauser and D. Wellmann, Hadronic Higgs boson decay at order $\alpha_{\mathrm{s}}^{4}$ and $\alpha_{\mathrm{s}}^{5}$, PoS (DIS2017) 295 [arXiv: 1706.00624] [INSPIRE].

[17] A. Djouadi, M. Spira and P.M. Zerwas, Production of Higgs bosons in proton colliders: QCD corrections, Phys. Lett. B 264 (1991) 440 [INSPIRE].

[18] K.G. Chetyrkin, B.A. Kniehl and M. Steinhauser, Hadronic Higgs decay to order $\alpha_{\mathrm{s}}^{4}$, Phys. Rev. Lett. 79 (1997) 353 [hep-ph/9705240] [INSPIRE].

[19] P.A. Baikov, K.G. Chetyrkin and J.H. Kuhn, Order $\alpha_{\mathrm{s}}^{4} Q C D$ Corrections to $Z$ and tau Decays, Phys. Rev. Lett. 101 (2008) 012002 [arXiv:0801.1821] [INSPIRE].

[20] P.A. Baikov, K.G. Chetyrkin and J.H. Kühn, Adler Function, Bjorken Sum Rule and the Crewther Relation to Order $\alpha_{\mathrm{s}}^{4}$ in a General Gauge Theory, Phys. Rev. Lett. 104 (2010) 132004 [arXiv:1001.3606] [InSPIRE].

[21] P.A. Baikov, K.G. Chetyrkin, J.H. Kühn and J. Rittinger, Complete $\mathcal{O}\left(\alpha_{\mathrm{s}}^{4}\right) Q C D$ Corrections to Hadronic Z-Decays, Phys. Rev. Lett. 108 (2012) 222003 [arXiv:1201.5804] [INSPIRE].

[22] P.A. Baikov, K.G. Chetyrkin, J.H. Kühn and J. Rittinger, $R(s)$ and $Z$ decay in order $\alpha_{\mathrm{s}}^{4}$ : complete results, PoS (RADCOR2011) 030 [arXiv:1210.3594] [INSPIRE].

[23] P.A. Baikov, K.G. Chetyrkin and J.H. Kühn, Massless Propagators, R(s) and Multiloop QCD, Nucl. Part. Phys. Proc. 261-262 (2015) 3 [arXiv:1501.06739] [inSPIRE].

[24] C.G. Bollini and J.J. Giambiagi, Dimensional Renormalization: The Number of Dimensions as a Regularizing Parameter, Nuovo Cim. 12B (1972) 20.

[25] G. 't Hooft and M.J.G. Veltman, Regularization and Renormalization of Gauge Fields, Nucl. Phys. B 44 (1972) 189 [inSPIRE]. 
[26] T. Ueda, B. Ruijl and J.A.M. Vermaseren, Calculating four-loop massless propagators with Forcer, J. Phys. Conf. Ser. 762 (2016) 012060 [arXiv: 1604. 08767] [INSPIRE].

[27] T. Ueda, B. Ruijl and J.A.M. Vermaseren, Forcer: a FORM program for 4-loop massless propagators, PoS (LL2016) 070 [arXiv: 1607.07318] [INSPIRE].

[28] B. Ruijl, T. Ueda and J.A.M. Vermaseren, Forcer, a FORM program for the parametric reduction of four-loop massless propagator diagrams, arXiv:1704.06650 [INSPIRE].

[29] J.A.M. Vermaseren, New features of FORM, math-ph/0010025 [INSPIRE].

[30] M. Tentyukov and J.A.M. Vermaseren, The Multithreaded version of FORM, Comput. Phys. Commun. 181 (2010) 1419 [hep-ph/0702279] [INSPIRE].

[31] J. Kuipers, T. Ueda, J.A.M. Vermaseren and J. Vollinga, FORM version 4.0, Comput. Phys. Commun. 184 (2013) 1453 [arXiv:1203.6543] [INSPIRE].

[32] F. Herzog and B. Ruijl, The $R^{*}$-operation for Feynman graphs with generic numerators, JHEP 05 (2017) 037 [arXiv:1703.03776] [INSPIRE].

[33] K.G. Chetyrkin and F.V. Tkachov, Infrared $R$ operation and ultraviolet counterterms in the MS scheme, Phys. Lett. 114B (1982) 340 [INSPIRE].

[34] K.G. Chetyrkin and V.A. Smirnov, $R^{*}$ operation corrected, Phys. Lett. 144B (1984) 419 [INSPIRE].

[35] D.V. Batkovich and M. Kompaniets, Toolbox for multiloop Feynman diagrams calculations using $R^{*}$ operation, J. Phys. Conf. Ser. 608 (2015) 012068 [arXiv:1411.2618] [INSPIRE].

[36] D.V. Batkovich, K.G. Chetyrkin and M.V. Kompaniets, Six loop analytical calculation of the field anomalous dimension and the critical exponent $\eta$ in $O(n)$-symmetric $\varphi^{4}$ model, Nucl. Phys. B 906 (2016) 147 [arXiv: 1601.01960] [INSPIRE].

[37] K.G. Chetyrkin, Combinatorics of $R-, R^{-1}$ - and $R^{*}$-operations and asymptotic expansions of Feynman integrals in the limit of large momenta and masses, MPI-PH-PTH-13-91 (1991).

[38] P.A. Baikov, K.G. Chetyrkin and J.H. Kühn, Five-Loop Running of the QCD coupling constant, Phys. Rev. Lett. 118 (2017) 082002 [arXiv:1606. 08659] [INSPIRE].

[39] F. Herzog, B. Ruijl, T. Ueda, J.A.M. Vermaseren and A. Vogt, The five-loop $\beta$-function of Yang-Mills theory with fermions, JHEP 02 (2017) 090 [arXiv: 1701.01404] [INSPIRE].

[40] G. 't Hooft, Dimensional regularization and the renormalization group, Nucl. Phys. B 61 (1973) 455 [INSPIRE].

[41] W.A. Bardeen, A.J. Buras, D.W. Duke and T. Muta, Deep Inelastic Scattering Beyond the Leading Order in Asymptotically Free Gauge Theories, Phys. Rev. D 18 (1978) 3998 [INSPIRE].

[42] L. von Smekal, K. Maltman and A. Sternbeck, The Strong coupling and its running to four loops in a minimal MOM scheme, Phys. Lett. B 681 (2009) 336 [arXiv:0903.1696] [INSPIRE].

[43] J.A. Gracey, Renormalization group functions of QCD in the minimal MOM scheme, J. Phys. A 46 (2013) 225403 [arXiv: 1304.5347] [inSPIRE].

[44] B. Ruijl, T. Ueda, J.A.M. Vermaseren and A. Vogt, Four-loop QCD propagators and vertices with one vanishing external momentum, JHEP 06 (2017) 040 [arXiv:1703. 08532] [INSPIRE]. 
[45] D.-M. Zeng, S.-Q. Wang, X.-G. Wu and J.-M. Shen, The Higgs-boson decay $H \rightarrow$ gg up to $\alpha_{\mathrm{s}}^{5}$-order under the minimal momentum space subtraction scheme,

J. Phys. G 43 (2016) 075001 [arXiv: 1507.03222] [INSPIRE].

[46] K.G. Chetyrkin and M. Steinhauser, The Relation between the MS-bar and the on-shell quark mass at order $\alpha_{\mathrm{s}}^{3}$, Nucl. Phys. B 573 (2000) 617 [hep-ph/9911434] [INSPIRE].

[47] K. Melnikov and T.v. Ritbergen, The Three loop relation between the MS-bar and the pole quark masses, Phys. Lett. B 482 (2000) 99 [hep-ph/9912391] [INSPIRE].

[48] P. Nogueira, Automatic Feynman graph generation, J. Comput. Phys. 105 (1993) 279 [INSPIRE].

[49] T. van Ritbergen, A.N. Schellekens and J.A.M. Vermaseren, Group theory factors for Feynman diagrams, Int. J. Mod. Phys. A 14 (1999) 41 [hep-ph/9802376] [INSPIRE].

[50] F. Herzog, B. Ruijl, T. Ueda, J.A.M. Vermaseren and A. Vogt, FORM, Diagrams and Topologies, PoS (LL2016) 073 [arXiv: 1608.01834] [INSPIRE].

[51] W.L. van Neerven and A. Vogt, Nonsinglet structure functions beyond the next-to-next-to-leading order, Nucl. Phys. B 603 (2001) 42 [hep-ph/0103123] [InSPIRE].

[52] T. van Ritbergen, J.A.M. Vermaseren and S.A. Larin, The Four loop $\beta$-function in quantum chromodynamics, Phys. Lett. B 400 (1997) 379 [hep-ph/9701390] [INSPIRE].

[53] M. Czakon, The four-loop QCD $\beta$-function and anomalous dimensions, Nucl. Phys. B 710 (2005) 485 [hep-ph/0411261] [INSPIRE].

[54] S. Alekhin, J. Blümlein, S. Moch and R. Placakyte, Parton Distribution Functions, $\alpha_{\mathrm{s}}$ and Heavy-Quark Masses for LHC Run II, Phys. Rev. D 96 (2017) 014011 [arXiv:1701.05838] [INSPIRE].

[55] Particle Data Group collaboration, C. Patrignani et al., Review of Particle Physics, Chin. Phys. C 40 (2016) 100001.

[56] S.G. Gorishnii, A.L. Kataev, S.A. Larin and L.R. Surguladze, Corrected Three Loop QCD Correction to the Correlator of the Quark Scalar Currents and $\Gamma_{\text {tot }}\left(H^{0} \rightarrow\right.$ Hadrons), Mod. Phys. Lett. A 5 (1990) 2703 [InSPIRE].

[57] K.G. Chetyrkin, Correlator of the quark scalar currents and Gamma(tot) ( $H \rightarrow$ hadrons) at $O\left(\alpha_{\mathrm{s}}^{3}\right)$ in pQCD, Phys. Lett. B 390 (1997) 309 [hep-ph/9608318] [INSPIRE].

[58] K.G. Chetyrkin, Quark mass anomalous dimension to $O\left(\alpha_{\mathrm{s}}^{4}\right)$, Phys. Lett. B 404 (1997) 161 [hep-ph/9703278] [INSPIRE].

[59] J.A.M. Vermaseren, S.A. Larin and T. van Ritbergen, The four loop quark mass anomalous dimension and the invariant quark mass, Phys. Lett. B 405 (1997) 327 [hep-ph/9703284] [INSPIRE].

[60] S.G. Gorishnii, A.L. Kataev and S.A. Larin, The $O\left(\alpha_{\mathrm{s}}^{3}\right)$-corrections to $\sigma_{\text {tot }}\left(e^{+} e^{-} \rightarrow\right.$ hadrons $)$ and $\Gamma\left(\tau^{-} \rightarrow \nu_{\tau}+\right.$ hadrons) in QCD, Phys. Lett. B 259 (1991) 144 [INSPIRE].

[61] L.R. Surguladze and M.A. Samuel, Total hadronic cross-section in $e^{+} e^{-}$annihilation at the four loop level of perturbative QCD, Phys. Rev. Lett. 66 (1991) 560 [Erratum ibid. 66 (1991) 2416] [INSPIRE].

[62] S. Moch, J.A.M. Vermaseren and A. Vogt, On $\gamma_{5}$ in higher-order QCD calculations and the NNLO evolution of the polarized valence distribution, Phys. Lett. B 748 (2015) 432 [arXiv: 1506.04517] [INSPIRE]. 
[63] J.A. Gracey, Momentum subtraction and the $R$ ratio, Phys. Rev. D 90 (2014) 094026 [arXiv: 1410.6715] [INSPIRE].

[64] A.L. Kataev and V.S. Molokoedov, Fourth-order QCD renormalization group quantities in the $V$ scheme and the relation of the $\beta$ function to the Gell-Mann-Low function in QED, Phys. Rev. D 92 (2015) 054008 [arXiv: 1507.03547] [InSPIRE].

[65] K. Fujii et al., Physics Case for the International Linear Collider, arXiv:1506.05992 [INSPIRE].

[66] J.C. Collins and J.A.M. Vermaseren, Axodraw Version 2, arXiv:1606.01177 [InSPIRE]. 